CRYSTALLOGRAPHIC COMMUNICATIONS

ISSN 2056-9890

Received 5 August 2015

Accepted 28 October 2015

Edited by A. J. Lough, University of Toronto, Canada

Keywords: crystal structure; copper; dihydroxypyridine; potential water oxidation catalysts

CCDC references: $1433678 ; 1000450$ Supporting information: this article has supporting information at journals.iucr.org/e

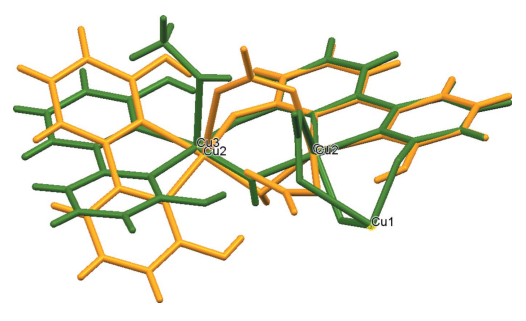

OPEN $\odot$ ACCESS

\section{Crystal structures of bis- and hexakis[(6,6'-di- hydroxybipyridine)copper(II)] nitrate coordination complexes}

\author{
Deidra L. Gerlach, ${ }^{\text {a Ismael Nieto, }}{ }^{\text {b }}$ Corey J. Herbst-Gervasoni, ${ }^{\text {b }}$ Gregory M. \\ Ferrence, ${ }^{\mathrm{c}}$ Matthias Zeller ${ }^{\mathrm{d}}$ and Elizabeth T. Papish ${ }^{\mathrm{a} *}$
}

a Department of Chemistry, University of Alabama, 250 Hackberry Lane, Tuscaloosa, AL 35487-0336, USA, ${ }^{\mathbf{b}}$ Department of Chemistry, Drexel University, 3141 Chestnut St., Philadelphia, PA 19104, USA, ' Department of Chemistry, Illinois State University, Campus Box 4160, Normal, IL 61790-4160, USA, and department of Chemistry, Youngstown State University, One University Plaza, Youngstown, OH 44555, USA. *Correspondence e-mail: etpapish@ua.edu

Two multinuclear complexes synthesized from $\mathrm{Cu}\left(\mathrm{NO}_{3}\right)_{2}$ and $6,6^{\prime}$-dihydroxybipyridine (dhbp) exhibit bridging nitrate and hydroxide ligands. The dinuclear complex $\quad\left(6,6^{\prime}\right.$-dihydroxybipyridine- $\left.2 \kappa^{2} N, N^{\prime}\right)[\mu-6-(6-h y d r o x y p y r i d i n-2-y l)$ -

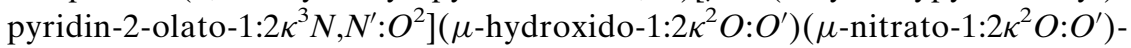

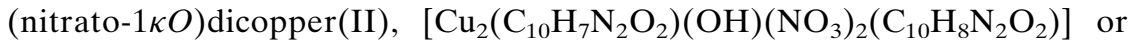
$\left[\mathrm{Cu}\left(6-\mathrm{OH}-6^{\prime}-\mathrm{O}-\mathrm{bpy}\right)\left(\mathrm{NO}_{3}\right)(\mu-\mathrm{OH})\left(\mu-\mathrm{NO}_{3}\right) \mathrm{Cu}\left(6,6^{\prime}-\mathrm{dhbp}\right)\right]$, (I), with a $2: 1$ ratio of nitrate to hydroxide anions and one partially deprotonated dhbp ligand, forms from a water-ethanol mixture at neutral $\mathrm{pH}$. The hexanuclear complex bis $\left(\mu_{3}\right.$-bipyridine-2,2'-diolato- $\left.\kappa^{3} O: N, N^{\prime}: O^{\prime}\right)$ tetrakis $\left(6,6^{\prime}\right.$-dihydroxybipyridine$\left.\kappa^{2} N, N^{\prime}\right)$ tetrakis $\left(\mu\right.$-hydroxido- $\left.\kappa^{2} O: O^{\prime}\right)$ bis (methanol- $\left.\kappa O\right)$ tetrakis $\left(\mu\right.$-nitrato- $\left.\kappa^{2} O: O^{\prime}\right)$ hexacopper(II), $\left[\mathrm{Cu}_{6}\left(\mathrm{C}_{10} \mathrm{H}_{6} \mathrm{~N}_{2} \mathrm{O}_{2}\right)_{2}\left(\mathrm{CH}_{4} \mathrm{O}\right)_{2}(\mathrm{OH})_{4}\left(\mathrm{NO}_{3}\right)_{4}\left(\mathrm{C}_{10} \mathrm{H}_{8} \mathrm{~N}_{2} \mathrm{O}_{2}\right)_{4}\right]$ or $\left[\mathrm{Cu}\left(6,6^{\prime}-\mathrm{dhbp}\right)\left(\mu-\mathrm{NO}_{3}\right)_{2}(\mu-\mathrm{OH}) \mathrm{Cu}\left(6,6^{\prime}-\mathrm{O}-\mathrm{bpy}\right)(\mu-\mathrm{OH}) \mathrm{Cu}\left(6,6^{\prime} \mathrm{dhbp}\right)\left(\mathrm{CH}_{3} \mathrm{OH}\right)\right]_{2}$, (II), with a 1:1 $\mathrm{NO}_{3}-\mathrm{OH}$ ratio and two fully protonated and fully deprotonated dhbp ligands, was obtained by methanol recrystallization of material obtained at pH 3. Complex (II) lies across an inversion center. Complexes (I) and (II) both display intramolecular $\mathrm{O}-\mathrm{H} \cdots \mathrm{O}$ hydrogen bonding. Intermolecular $\mathrm{O}-\mathrm{H} \cdots \mathrm{O}$ hydrogen bonding links symmetry-related molecules forming chains along [100] for complex (I) with $\pi$-stacking along [010] and [001]. Complex (II) forms intermolecular $\mathrm{O}-\mathrm{H} \cdots \mathrm{O}$ hydrogen-bonded chains along [010] with $\pi$-stacking along [100] and [001].

\section{Chemical context}

Catalytic processes in nature are often facilitated by enzymes that feature transition metals in their active sites. Many of these reactions would be of tremendous interest could they be copied using simpler and technologically feasible conditions. One such process is water oxidation as observed in photosynthesis, and the use of transition metal complexes to mimic the reactivity of photosystem II have captured the attention of an increasing number of research groups over the last few years (Kikuchi \& Tanaka, 2014; Singh \& Spiccia, 2013). One complex that especially caught our interest was $[\mathrm{Cp} * \operatorname{Ir}(\text { bpy }) \mathrm{Cl}]^{+}$(Blakemore et al., 2010) which features a bipyridine (bpy) type ligand. In our research into catalytic water oxidation, we are trying to enhance proton-coupled electron transfer (PCET) in metal-complex catalysts by incorporating hydrogen-bond donors and acceptors in near proximity to the potentially catalytic metal atoms to mimic the active center of a protein-metal complex. When applying this principal to the Blakemore-type $[\mathrm{Cp} * \operatorname{Ir}(\mathrm{bpy}) \mathrm{Cl}]^{+}$complex by swapping normal bipyridine for dihydroxybipyridine (6,6'dhbp), we were indeed able to increase the catalytic turnover 
rate and control water oxidation rates by adjusting $\mathrm{pH}$ levels (DePasquale et al., 2013). The ligand 6,6'-dhbp has also been used in combination with ruthenium terpyridine (tpy) fragments to yield the complex $\left[(\right.$ tpy $\left.) \mathrm{Ru}\left(6,6^{\prime}-\mathrm{dhbp}\right)\left(\mathrm{H}_{2} \mathrm{O}\right)\right]$ (Marelius et al., 2014).

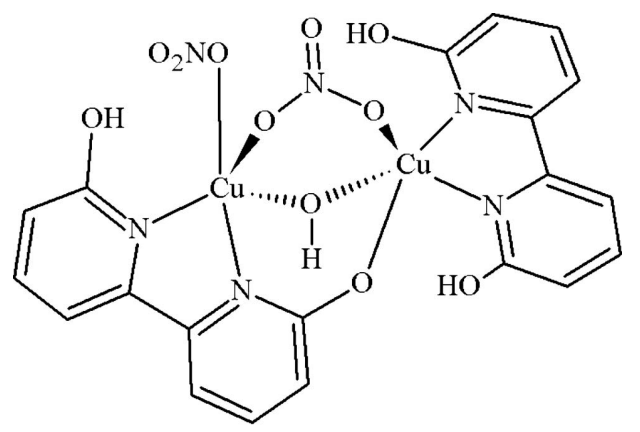

(I)

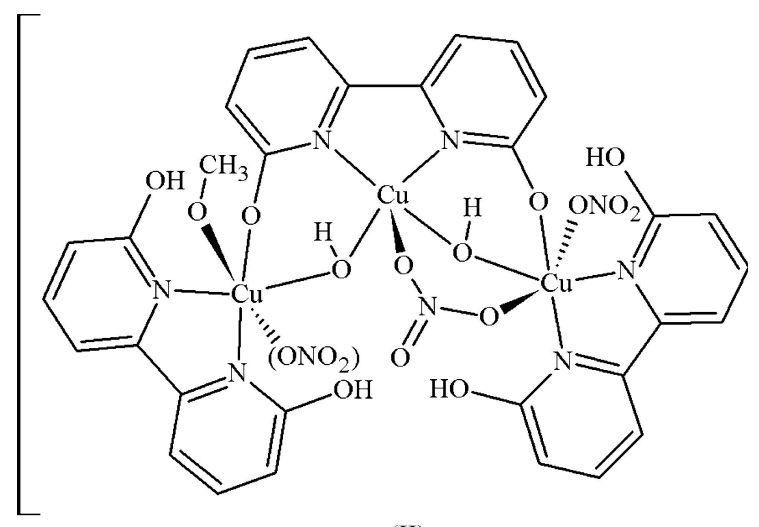

(II)

Our focus has most recently shifted to the investigation of copper(II) bipyridine complexes analogous to $\left[(\text { bpy }) \mathrm{Cu}(\mathrm{OH})_{2}\right]_{2}{ }^{2+}$ (Barnett et al., 2012). We isolated discrete mono-copper complexes from copper(II) sulfate with a selection of modified bipyridine ligands and investigated the compounds spectroscopically, crystallographically and for their catalytic water oxidation capacity (Gerlach et al., 2014). When swapping sulfate for nitrate as the counter-ion we found that the resulting complexes are no longer mononuclear. Instead, larger aggregates with two or six copper(II) atoms formed that feature coordinating nitrate as well as hydroxyl ligands. As a result of their aggregation and the varied coordination environment of their copper atoms, these complexes are not ideally suited for homogenous water oxidation catalysis. Instead they feature quite intriguing and fascinating solid structures which we would like to describe and present.

\section{Structural commentary}

The dinuclear copper(II) dhbp complex (I) contains nitrate as a co-ligand with both a fully protonated and a mono-deprotonated dhbp ligand (see Fig. 1). Two unique 6,6'-dhbp binding modes were observed for this copper(II) nitrate complex illustrating the structural flexibility of this ligand. The
Table 1

Hydrogen-bond geometry $\left(\AA,^{\circ}\right)$ for (I).

\begin{tabular}{|c|c|c|c|c|}
\hline$D-\mathrm{H} \cdots A$ & $D-\mathrm{H}$ & $\mathrm{H} \cdots A$ & $D \cdots A$ & $D-\mathrm{H} \cdots A$ \\
\hline $\mathrm{O} 5-\mathrm{H} 15 \cdots \mathrm{O} 11^{\mathrm{i}}$ & $0.75(4)$ & $2.08(4)$ & $2.802(4)$ & $164(4)$ \\
\hline $\mathrm{O} 1-\mathrm{H} 1 \cdots \mathrm{O} 6$ & $0.83(2)$ & $1.78(2)$ & $2.583(4)$ & $163(5)$ \\
\hline $\mathrm{O} 3-\mathrm{H} 3 B \cdots \mathrm{O} 5$ & $0.83(2)$ & $1.70(2)$ & $2.510(4)$ & $164(5)$ \\
\hline $\mathrm{O} 4-\mathrm{H} 4 B \cdots \mathrm{O} 2$ & $0.83(2)$ & $1.73(2)$ & $2.528(4)$ & $161(5)$ \\
\hline
\end{tabular}

Symmetry code: (i) $x+1, y, z$.

$\left[6,6^{\prime}-(\mathrm{OH})_{2}\right.$ bpy $]$ ligand exhibits the typically observed bipyridine $(N, N)$ coordination mode through $\mathrm{N} 3$ and $\mathrm{N} 4$ binding to $\mathrm{Cu} 2$. A new coordination mode is observed for the monodeprotonated (6-O-6'-OH-bpy) ligand in which it bridges two metals, through $(N, N)$ coordination of $\mathrm{N} 1, \mathrm{~N} 2$ to $\mathrm{Cu} 1$ and through bridging to $\mathrm{Cu} 2$ via the pyridinolate oxygen $\mathrm{O} 2$ [1.946 (3) §]. The C-O bond lengths ( $\mathrm{A})$ for the dhbp ligands are $1.335(5)(\mathrm{C} 1-\mathrm{O} 1), 1.322(5)(\mathrm{C} 11-\mathrm{O} 3)$, and $1.316(4)$ (C20-O4) for the protonated hydroxyl groups and slightly shorter at $1.310(4)(\mathrm{C} 10-\mathrm{O} 2)$ for the pyridinolate, reflecting double-bond character. Both copper atoms have a distorted square-pyramidal geometry with $\tau=0.394$ at $\mathrm{Cu} 1$ and $\tau=0.119$ at $\mathrm{Cu} 2$ (Addison et al., 1984). This structure has a close $\mathrm{Cu} \cdots \mathrm{Cu}$ distance of 3.158 (9) А. Complex (I) features three strong intramolecular hydrogen bonds (Jeffrey, 2003) with O...O distances ( $\mathrm{A}$ ) as follows: 2.583 (4) for O1 to O6 (dhbp to nitrate) with bond angle $\mathrm{O}-\mathrm{H} \cdots \mathrm{O}$ of $163(5)^{\circ}, 2.528$ (4) for $\mathrm{O} 4$ to $\mathrm{O} 2$ (dhbp to deprotonated dhbp) with bond angle of $161(5)^{\circ}$, and 2.510 (4) for O3 to O5 (dhbp to hydroxyl) with bond angle of $164(5)^{\circ}$. One intermolecular hydrogen bond tethers one dimer of complex (I) to the next in a head-to-tail fashion via a 2.802 (4) $\AA$ hydrogen bond from O5 to O11 (hydroxyl to nitrate) with a bond angle of $164(4)^{\circ}$. Numerical details of the hydrogen bonds are given in Table 1 .

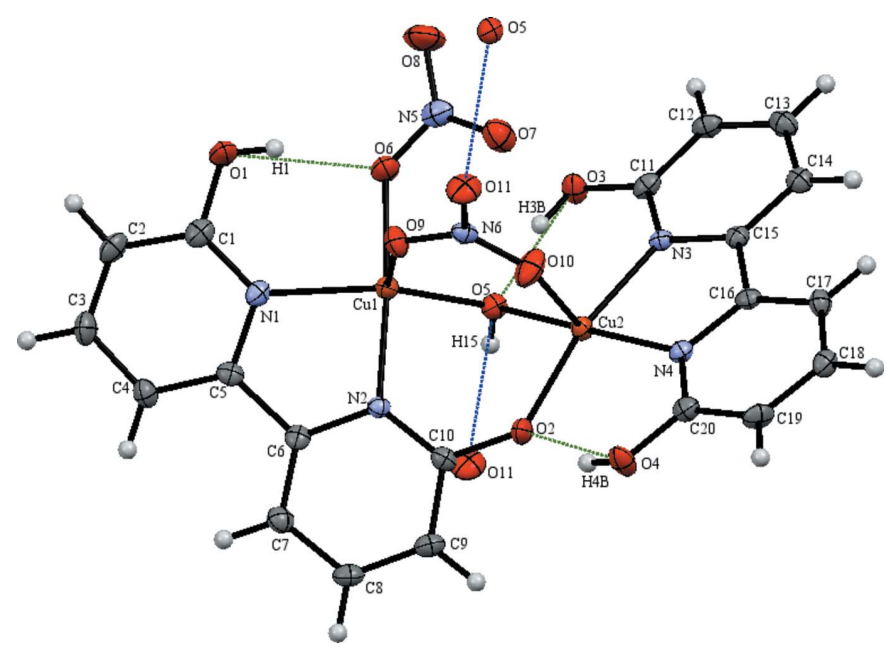

Figure 1

The numbering scheme of complex (I), with donor-acceptor distances of intramolecular hydrogen bonds colored green and of intermolecular hydrogen bonds colored blue, represented with displacement ellipsoids at the $50 \%$ probability level. Additional symmetry-related atoms O5 and O11 were generated by translation along the $a$ axis. 

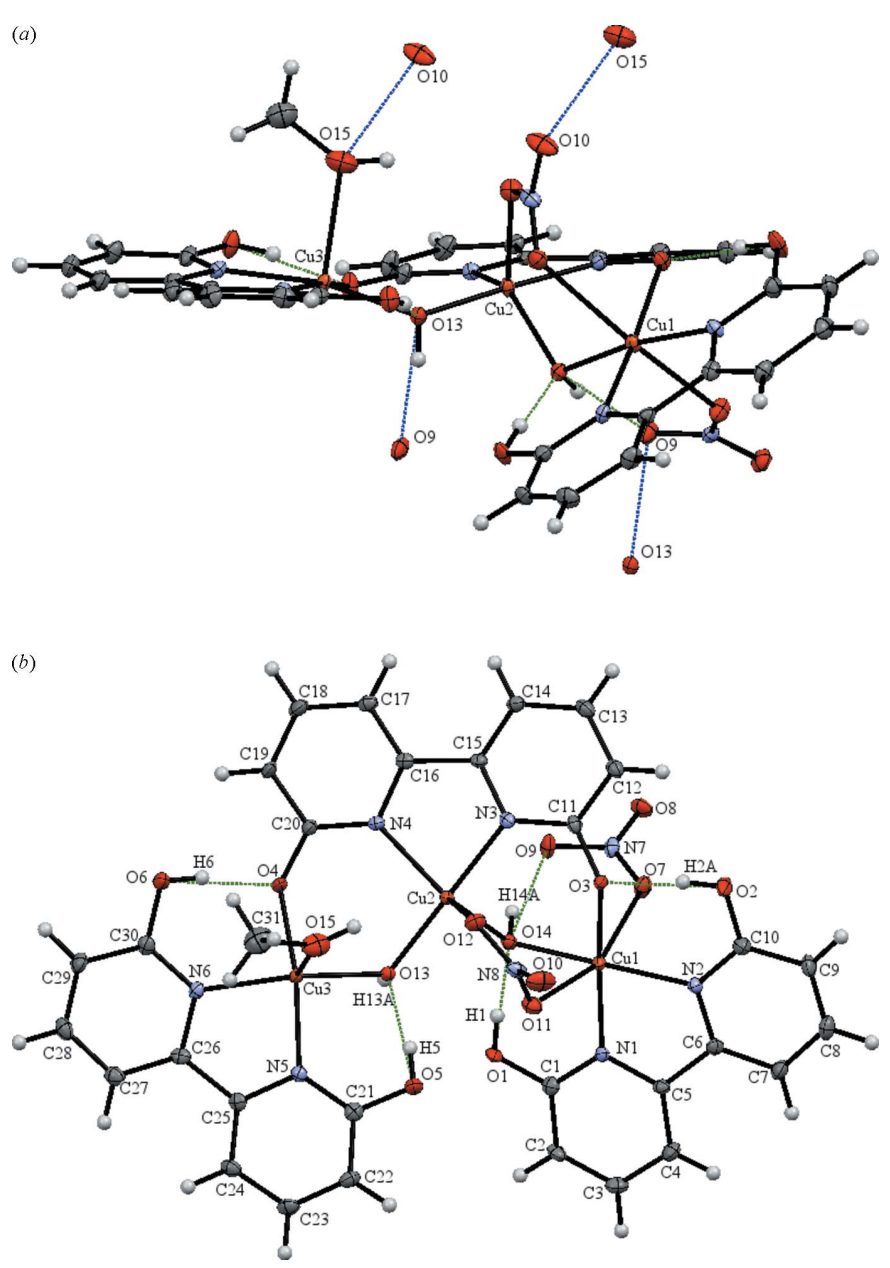

Figure 2

(a) The asymmetric unit of complex (II) represented with displacement ellipsoids at the $50 \%$ probability level. H atoms not involved in hydrogen bonding are omitted for clarity. The donor-acceptor distances are shown as green for intramolecular hydrogen bonds and shown as blue for intermolecular hydrogen bonds $(\mathrm{O} 10-\mathrm{O} 15)$ and inter-asymmetric unit hydrogen bonds (O9-O13). Additional symmetry-related atoms $\mathrm{O} 10$ and $\mathrm{O} 15$ were generated by the symmetry operator $1-x,-y, 1-z$, and $\mathrm{O} 9$ and $\mathrm{O} 13$ were generated via the inversion center $1-x, 1-y, 1-z$ at the center of the hexanuclear complex. (b) The asymmetric unit of complex (II) oriented to show donor-acceptor distances of intramolecular hydrogen bonds, in green, of the protonated dhbp ligands.

The hexanuclear copper(II) dhbp complex (II) is comprised of a dimer of the asymmetric portion of the molecule which contains three symmetry-unique copper atoms, two fully protonated and one fully deprotonated dhbp, two bridging hydroxide and two nitrate ligands (see Fig. 2). This asymmetric trinuclear unit is related through an inversion center to the full hexanuclear complex (see Fig. 3). Two copper atoms, Cu1 and $\mathrm{Cu} 3$, are hexa-coordinate with a distorted octahedral geometry whereas $\mathrm{Cu} 2$ is penta-coordinate with a distorted trigonal-pyramidal geometry with $\tau=0.746$ (Addison et al., 1984). Similar to the dinuclear complex, each copper atom is coordinated by one hydroxybipyridine ligand with one bridging hydroxyl ligand between $\mathrm{Cu} 1$ to $\mathrm{Cu} 2$ and $\mathrm{Cu} 2$ to $\mathrm{Cu} 3$. The dihydroxybipyridine ligand bound to $\mathrm{Cu} 2(\mathrm{dhbp} 2)$ is doubly deprotonated with each deprotonated oxygen bound to the

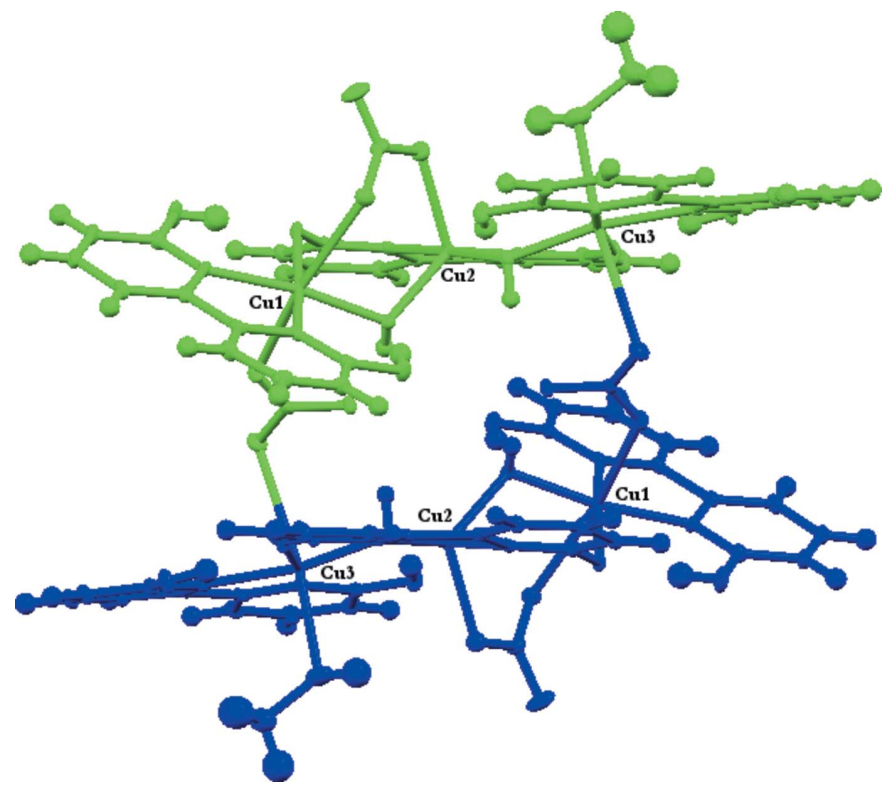

Figure 3

The full hexanuclear complex (II) represented with displacement ellipsoids at the $50 \%$ probability level and $\mathrm{H}$ atoms not involved in hydrogen bonding are omitted for clarity. The two symmetry-related units of the hexamer are shown in blue and green to better visualize the relation of the asymmetric unit through the inversion center.

flanking $\mathrm{Cu} 1$ and $\mathrm{Cu} 3$ metal sites, $\mathrm{O} 3$ and $\mathrm{O} 4$, respectively. The remaining coordination sphere of $\mathrm{Cu} 1$ entails one $\operatorname{dhbp}(N, N$ bound), one bridging hydroxide to $\mathrm{Cu} 2$ (O14), one bridging nitrate to $\mathrm{Cu} 2$ (O11), and one bridging nitrate (O7) which tethers the two asymmetric units. The coordination of $\mathrm{Cu} 2$ entails one deprotonated dhbp ( $N, N$ bound), one bridging nitrate to $\mathrm{Cu} 1$ (O12), and two bridging hydroxides to $\mathrm{Cu} 1$ and $\mathrm{Cu} 3$ (O14 and O13, respectively). The remaining coordination sphere of $\mathrm{Cu} 3$ entails one dhbp ( $N, N$ bound), one methanol (O15), one bridging hydroxide (O13), and one bridging nitrate

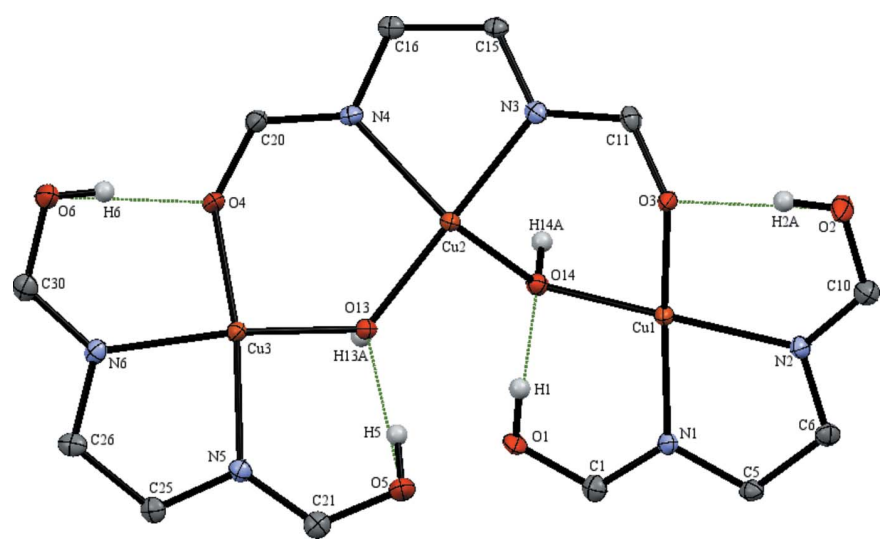

Figure 4

The three unique copper atoms of complex (II) are displayed with the coordination relevant atoms of the bound dhbp ligands and bridging hydroxides with displacement ellipsoids at the $50 \%$ probability level and the donor-acceptor distances of intramolecular hydrogen bonds of the protonated dhbps represented in green. 
Table 2

Hydrogen-bond geometry $\left(\AA,^{\circ}\right)$ for (II).

\begin{tabular}{|c|c|c|c|c|}
\hline$D-\mathrm{H} \cdots A$ & $D-\mathrm{H}$ & $\mathrm{H} \cdots A$ & $D \cdots A$ & $D-\mathrm{H} \cdots A$ \\
\hline $\mathrm{O} 1-\mathrm{H} 1 \cdots \mathrm{O} 14$ & 0.84 & 1.61 & $2.448(3)$ & 175 \\
\hline $\mathrm{O} 2-\mathrm{H} 2 A \cdots \mathrm{O} 3$ & 0.84 & 1.66 & 2.499 (3) & 172 \\
\hline $\mathrm{O} 5-\mathrm{H} 5 \cdots \mathrm{O} 13$ & 0.84 & 1.70 & $2.536(3)$ & 170 \\
\hline $\mathrm{O} 6-\mathrm{H} 6 \cdots \mathrm{O} 4$ & 0.84 & 1.67 & $2.495(3)$ & 168 \\
\hline $\mathrm{O} 13-\mathrm{H} 13 A \cdots \mathrm{O} 9^{\mathrm{i}}$ & $0.83(2)$ & $1.98(2)$ & $2.763(3)$ & $158(4)$ \\
\hline $\mathrm{O} 14-\mathrm{H} 14 A \cdots \mathrm{O} 9$ & $0.82(2)$ & $1.94(2)$ & $2.738(3)$ & $163(4)$ \\
\hline $\mathrm{O} 15-\mathrm{H} 15 \cdots \mathrm{O} 10^{\mathrm{ii}}$ & $0.85(5)$ & $2.42(5)$ & $2.790(4)$ & $107(4)$ \\
\hline
\end{tabular}

Symmetry codes: (i) $-x+1,-y+1,-z+1$; (ii) $-x+1,-y,-z+1$.

(O8) which tethers the two asymmetric units. Each deprotonated oxygen of dhbp acts as an acceptor for intramolecular hydrogen bonds from the two protonated dhbp ligands, $\mathrm{O} 2$ to O3 at 2.499 (3) $\AA\left(\mathrm{O}-\mathrm{H} \cdots \mathrm{O}\right.$ bond angle of $\left.172^{\circ}\right)$, and $\mathrm{O} 6$ to $\mathrm{O} 4$ at $2.495(3) \AA\left(\mathrm{O}-\mathrm{H} \cdots \mathrm{O}\right.$ bond angle of $\left.168^{\circ}\right)$ shown in Fig. 4. The remaining hydroxyl groups of the protonated dhbps form strong hydrogen bonds to the bridging hydroxides where O1 donates to $\mathrm{O} 14[2.448$ (3) $\AA$, O $-\mathrm{H} \cdots \mathrm{O}$ bond angle of $175^{\circ}$ ] of the hydroxide bridging $\mathrm{Cu} 1$ and $\mathrm{Cu} 2$, and $\mathrm{O} 5$ donates to $\mathrm{O} 13\left[2.536\right.$ (3) $\AA, \mathrm{O}-\mathrm{H} \cdots \mathrm{O}$ bond angle of $\left.170^{\circ}\right]$ of the hydroxide bridging $\mathrm{Cu} 2$ and $\mathrm{Cu} 3$ (Table 2). Interestingly, both $\mathrm{Cu}-\mathrm{Cu}$ bridging hydroxides form an intermediate strength intramolecular hydrogen bond to the bridging nitrate linking the two asymmetric units of the hexamer, with $\mathrm{O} \cdots \mathrm{O}$ distances $(\AA)$ of $\mathrm{O} 13$ and $\mathrm{O} 14$ to O9 being 2.763 (3) [O$\mathrm{H} \cdots \mathrm{O}$ angle of $158(4)^{\circ}$ ] and $2.738(3)$ [O $-\mathrm{H} \cdots \mathrm{O}$ angle of $163(4)^{\circ}$ ], respectively. $\mathrm{C}-\mathrm{O}$ bond lengths of the protonated and deprotonated dhbp ligands of the hexa and dinuclear complexes are similar; C. .O distances $(\AA)$ are 1.302 (4) and 1.312 (4) for the copper coordinating oxygen atoms, and 1.324 (4), 1.304 (4), 1.330 (4), and 1.321 (4) for the hydroxyl O atoms, with the longer of the four values belonging to the hydroxyl groups hydrogen-bound to the neighboring deprotonated dhbp ligand, and the shorter two being associated with those hydrogen-bound to the bridging hydroxyl groups. These reduced lengths reflect increased $\mathrm{C}-\mathrm{O}$ double-bond character upon deprotonation. One intermolecular hydrogen bond of intermediate strength connects the bound methanol to the non-coordinating oxygen of the $\mathrm{Cu} 1-\mathrm{Cu} 2$ bridging nitrate of another molecule, O15 to O10 at 2.790 (4) $\AA$ [O $-\mathrm{H} \cdots \mathrm{O}$ bond angle of $107(4)^{\circ}$ ].

Comparison of complex (I) to the asymmetric component of complex (II) indicates some structural similarities, Fig. 5. The overall structure of complex (I) can be reasonably well overlaid with the dinuclear component of (II) including $\mathrm{Cu} 2$ and $\mathrm{Cu} 3$, with the main differences resulting from one nitrate ligand that is bridging between the two copper ions in complex (I) being rotated so that in complex (II) it instead bridges one of these copper ions to the third that has no counterpart in complex (I). The two copper ions that are bridged by a nitrate ion in complex (I) are thus not bridged in complex (II) (featuring a methanol molecule and a nitrate bridging to the third copper instead), leading to a larger distance between the copper ions in complex (II) and a different tilt angle of the fully protonated dhbp ligand (left dhbp in Fig. 5).

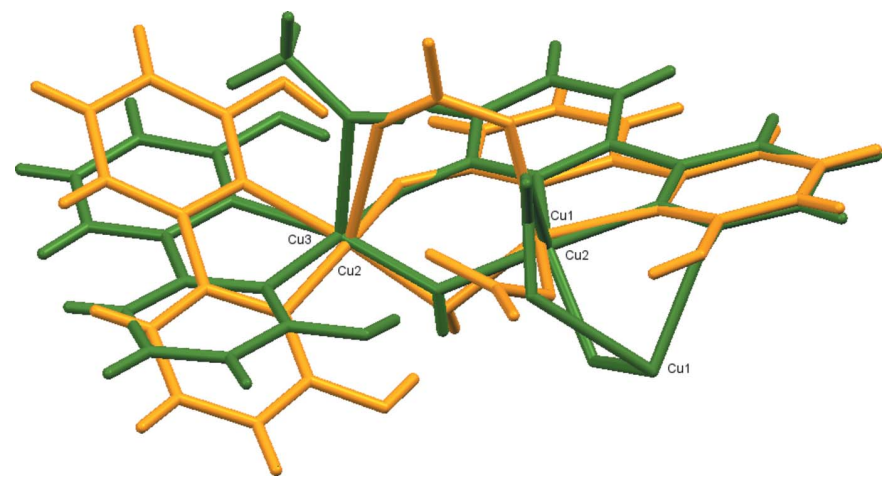

Figure 5

Complex (I) in yellow is overlaid on the $\mathrm{Cu} 2-\mathrm{Cu} 3$ dimer portion of the asymmetric unit of complex (II) in green.

The bridging oxygen species for both complexes (I) and (II) are correctly assigned as hydroxides to balance the overall neutral charge of the complex. Complex (I) with two $\mathrm{Cu}^{\mathrm{II}}$ ions is charge balanced with one terminal and one bridging nitrate each with a single negative charge, one deprotonated hydroxyl group of dhbp, and one bridging hydroxide. The bond lengths to the bridging hydroxide from Cu1 to O5 is 1.964 (3) $\AA$ and $\mathrm{Cu} 2$ to $\mathrm{O} 5$ is 1.939 (3) $\AA$ where the proton of O5 hydrogen bonds to one acceptor. A comparable bond length is 1.946 (3) $\AA$ from Cu2 to O2 of the deprotonated dhbp ligand. The remaining oxygen atoms of the dhbp ligands are each protonated and engaged in hydrogen bonding as described above. The asymmetric unit of complex (II) balances similarly with three $\mathrm{Cu}$ (II) ions against one bridging nitrate, one nitrate bridging the two asymmetric units, two deprotonated dhbp hydroxyl groups, and two bridging hydroxides. The bond lengths to the bridging hydroxide, $\mathrm{Cu} 2$ and $\mathrm{Cu} 3$ to $\mathrm{O} 13$ : 1.933 (2) and 1.951 (2) $\AA$, respectively, are comparable to those observed in complex (I) where each of these hydroxides have one hydrogen bond. Alternatively, the bond lengths to the bridging hydroxide are longer where $\mathrm{Cu} 1$ and $\mathrm{Cu} 2$ to $\mathrm{O} 14$ are 1.970 (2) and 2.062 (3) $\AA$, respectively, likely due to the two hydrogen-bonding interactions described above weakening the orbital overlap with the copper and lengthening these bonds. The two copper-hydroxyl dhbp bonds in complex (II) are comparable to this bond in complex (I) at 1.966 (3) $\AA$ from $\mathrm{Cu} 1$ to $\mathrm{O} 3$ of dhbp and 1.960 (2) $\AA$ from $\mathrm{Cu} 3$ to $\mathrm{O} 4$ of dhbp.

\section{Supramolecular features}

Some intermolecular hydrogen-bonding interactions in both complexes have already been discussed, vide supra. The dinuclear complex (I) also features intermolecular parallel offset $\pi$-stacking of both dhbp ligands. The dhbp ligand coordinating to $\mathrm{Cu} 1$ is $\pi$-stacked with its symmetry counterpart alternating across two inversion centers, one for each ring of this dhbp ligand. These alternating $\pi-\pi$ interactions form chains in the [010] direction. The pyridine ring containing N2 interacts with the symmetry-equivalent ring of a neighboring 


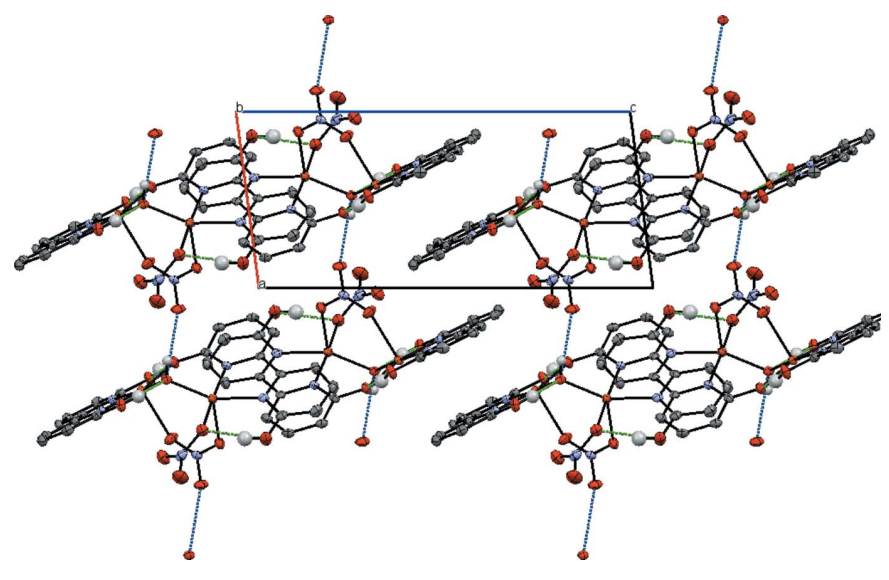

Figure 6

The packing arrangement of complex (I) propagates along [100] via intermolecular hydrogen bonding (blue) and in the $b c$ plane by $\pi$ stacking of the dhbp pyridyl rings.

molecule across the symmetry operation $1-x,-y,-z$ at a distance of 3.894 (3) A between the centroids of the rings. The pyridine ring containing $\mathrm{N} 1$ interacts with its symmetryequivalent ring across the symmetry operation $1-x, 1-y,-z$ with a centroid-to-centroid distance of 3.969 (3) $\AA$. The dhbp ligand coordinating to $\mathrm{Cu} 2$ also shows $\pi$-stacking via two alternating inversion-symmetry operations, forming chains along [100]. The pyridine rings containing N3 and N4 intercross by the symmetry operation $1-x,-y, 1-z$ where the centroid of the ring defined by $\mathrm{N} 3$ is at a distance of 3.604 (2) $\AA$ from the centroid of the ring defined as N4 on side of the dhbp plane with the bridging hydroxide. These rings also $\pi$-stack on the opposite face of the plane at a distance of 3.768 (2) $\AA$ from the centroid of the ring defined by N3 to N4 through the symmetry operation $-x,-y, 1-z$. Intermolecular hydrogen bonding from the bridging hydroxide ligand to the terminal oxygen of the bridging nitrate ligand interlinks neighboring molecules primarily along [100]. See Fig. 6 for extended intermolecular interactions of complex (I).

The hexanuclear complex (II) progresses along [010] through two symmetry-related hydrogen bonds between $\mathrm{O} 15$ of the bound methanol molecule of $\mathrm{Cu} 3$ to $\mathrm{O} 10$ of the $\mathrm{Cu} 1-$ $\mathrm{Cu} 2$ bridging nitrate (Fig. 7). The dhbp ligands are primarily within the $a c$ plane and exhibit $\pi$-stacking but in a less regular fashion than for complex (I), primarily in the [010] direction without forming chains. Off-set $\pi$-stacking of the dhbp ligand bound to $\mathrm{Cu} 1$ are related through the symmetry operation $1-x,-y,-z$ with a centroid-to-centroid distance of 3.784 (2) $\AA$ of the pyridine rings containing $\mathrm{N} 1$ to the ring containing $\mathrm{N} 2$ and vice versa. A single ring of each dhbp ligand bound to $\mathrm{Cu} 2$ and $\mathrm{Cu} 3 \pi$-stack via translation at a distance of 3.551 (2) $\AA$ between the centroids of the pyridine rings defined by $\mathrm{N} 3$ and N5, respectively. Additionally, the Cu3 dhbp ligand $\pi$-stacks via the symmetry-equivalent ring defined by $\mathrm{N} 5$ of a neighboring molecule across the symmetry operation $-x,-y, 1-z$ at a centroid-to-centroid distance of 3.887 (2) $\AA$. Close proximity occurs in plane between the pyridine ring containing $\mathrm{N} 4$ of the dhbp ligand bound to $\mathrm{Cu} 2$

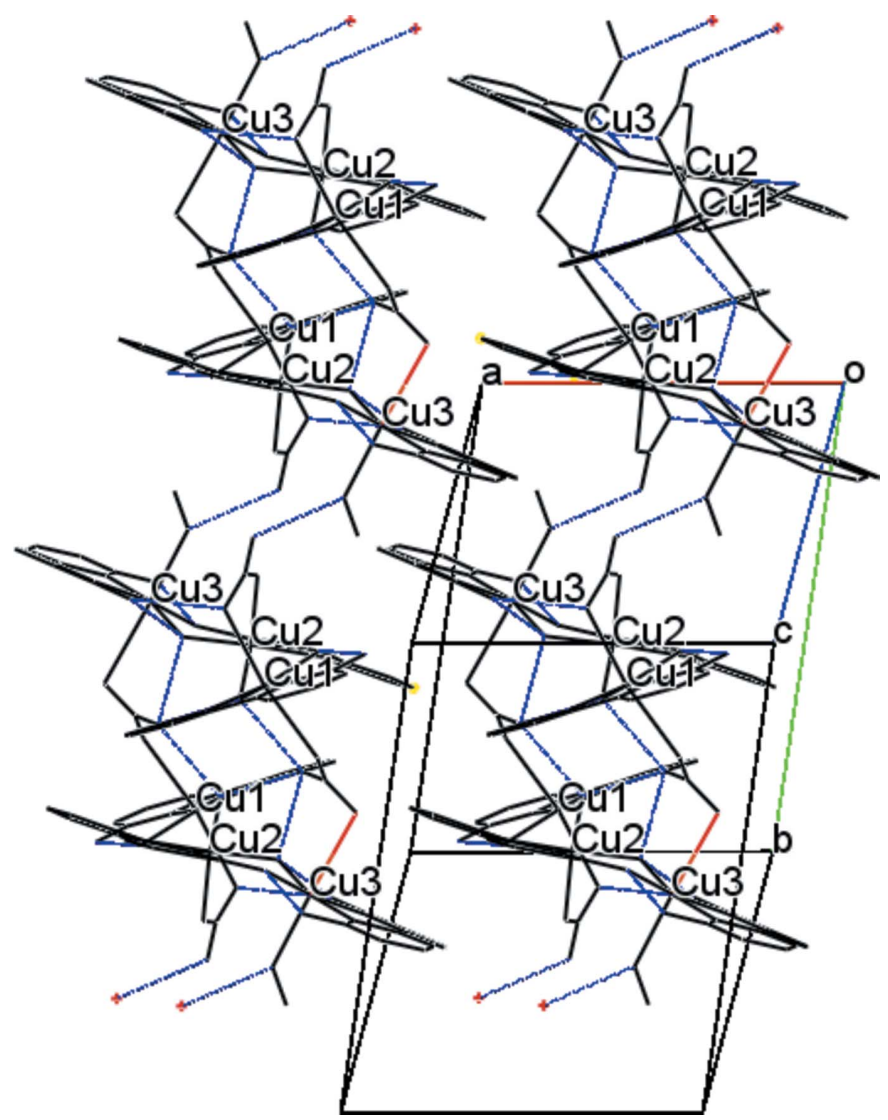

Figure 7

The packing arrangement of complex (II) propagates along [010] via intermolecular hydrogen bonding (blue) and in the $a c$ plane by $\pi$-stacking of the dhbp pyridyl rings.

at a distance of 3.818 (2) $\AA$ between C18 to C19 and vice versa across the symmetry operation $1-x, 1-y,-z$.

\section{Database survey}

Although many structures have been reported featuring a hydroxide anion bridging two copper(II) ions each bound by $2,2^{\prime}$-bipyridine, no analogous structure has been reported with a 6,6'-dihydroxy-2,2'-bipyridine ligand. A search of the Cambridge Structural Database (Version 5.36, May 2015; Groom \& Allen, 2014) for the substructure of copper ligated by 6-hydroxy-2,2'-bipyridine, where the hydroxyl group $(-\mathrm{OH})$ further ligates to a second copper atom, resulted in several structures. These primarily planar structures are reported either with co-crystallized metal-containing counterions: CSD refcode QEXHUX (Guo et al., 2007), VIHZIX (Zhong, Li et al., 2013), WUJGUE (Wang et al., 2009), XIQGAH (Zhong, Feng et al., 2013); or with a bridging molecule linking two of these planar copper dimers: IYOWOI (He \& Lu, 2004), MISPUZ (Zhang, Tong \& Chen, 2002), REMMAY (Luo et al., 2006), SESDAW (Sun et al., 2006), XOVTEH (Zhang, Tong, Gong et al., 2002).

The most relevant structure reported in the database contains a dinuclear copper 6-hydroxybipyridine complex with 
Table 3

Experimental details.

(I)

Crystal data

Chemical formula

$M_{\mathrm{r}}$

Crystal system, space group

Temperature (K)

$a, b, c(\AA)$

$\alpha, \beta, \gamma\left({ }^{\circ}\right)$

$V\left(\AA^{3}\right)$

$Z$

Radiation type

$\mu\left(\mathrm{mm}^{-1}\right)$

Crystal size (mm)

Data collection

Diffractometer

Absorption correction

$T_{\min }, T_{\max }$

No. of measured, independent and observed

$[I>2 \sigma(I)]$ reflections

$R_{\text {int }}$

$(\sin \theta / \lambda)_{\max }\left(\AA^{-1}\right)$

$\left[\mathrm{Cu}_{2}\left(\mathrm{C}_{10} \mathrm{H}_{7} \mathrm{~N}_{2} \mathrm{O}_{2}\right)(\mathrm{OH})\left(\mathrm{NO}_{3}\right)_{2}\left(\mathrm{C}_{10} \mathrm{H}_{8} \mathrm{~N}_{2} \mathrm{O}_{2}\right)\right]$

643.47

Triclinic, $P \overline{1}$

100

7.358 (2), 10.447 (3), 15.744 (4)

77.610 (4), 78.927 (4), 69.938 (4)

$1101.1(5)$

Mo $K \alpha$

2.01

$0.17 \times 0.12 \times 0.03$

Bruker SMART APEX CCD

Multi-scan (SADABS; Bruker, 2009)

$0.605,0.746$

$14887,6413,4302$

0.050

0.717

$0.052,0.125,1.04$

6413

365

3

$\mathrm{H}$ atoms treated by a mixture of independent and constrained refinement $1.11,-0.69$

$\Delta \rho_{\max }, \Delta \rho_{\min }\left(\mathrm{e} \AA^{-3}\right)$
(II)
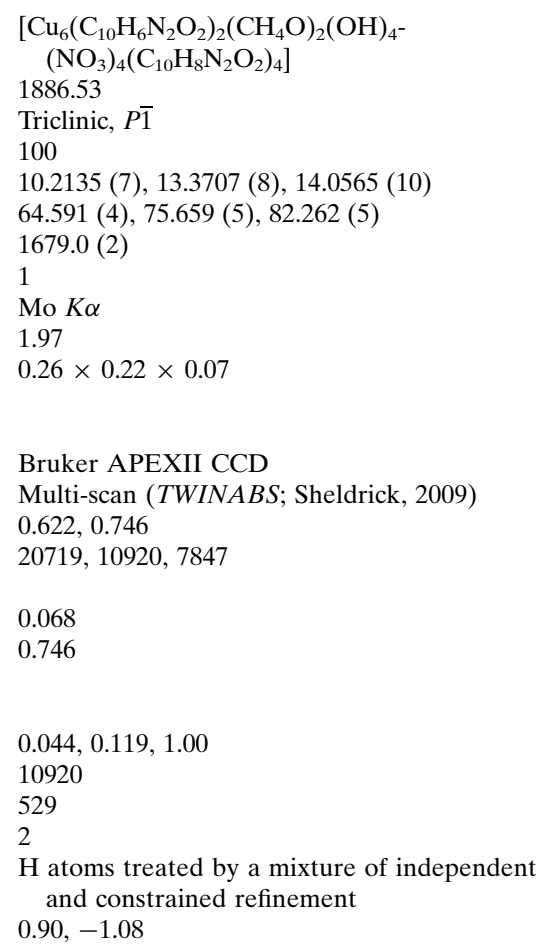

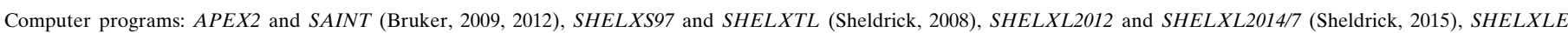
(Hübschle et al., 2011) and Mercury (Macrae et al., 2008).

a nitrate ligand bridging the copper ions, IBOXAZ (Zhang et al., 2004). No examples of hydroxybipyridine-ligated copper compounds with oxide or hydroxide bridges have been reported.

\section{Synthesis and crystallization}

The neutral copper dinuclear complex (I) Copper(II) nitrate trihydrate $(128 \mathrm{mg}, 0.530 \mathrm{mmol})$ and $6,6^{\prime}$-dhbp $(100 \mathrm{mg}$, $0.531 \mathrm{mmol}$ ) were combined in 50/50 ethanol and water solvent $(10 \mathrm{~mL})$ and stirred two days. Green plate crystals were grown from an ethanol solution in a freezer. This complex was analyzed exclusively by X-ray diffraction.

The neutral copper hexanuclear complex (II) Copper(II) nitrate hemipentahydrate (124 mg, $0.533 \mathrm{mmol})$ and 6,6'-dhbp $(100 \mathrm{mg}, 0.531 \mathrm{mmol})$ were combined in $10 \mathrm{~mL}$ of $0.1 \mathrm{M}$ $\mathrm{NaOAc}$ adjusted to $\mathrm{pH} 3$ by acetic acid. The mixture was stirred for three days at room temperature. The resulting solution was dried under high vacuum and recrystallized twice from methanol to afford green prismatic crystals. This complex was analyzed exclusively by x-ray diffraction.

\section{Refinement}

Crystal data, data collection, and structure refinement details are summarized in Table 3 .
The crystal under investigation for complex (II) was found to be split with two major domains not related by any obvious twin operation. The orientation matrices for the two components were identified using the program Cell Now (Sheldrick, 2008 ), with the two components being related by a $2.9^{\circ}$ rotation about either the reciprocal axis $1.000-0.363-0.339$ or the real axis $1.000-0.178-0.265$. The two components were integrated using SAINT (Bruker, 2012), resulting in the following statistics: 17535 data (5769 unique) involve domain 1 only, mean $I / \sigma 8.6,17271$ data (5689 unique) involve domain 2 only, mean $I / \sigma$ 8.2, 34813 data (9811 unique) involve 2 domains, mean I/sigma 9.5, 11 data (11 unique) involve 3 domains, mean $I / \sigma 8.7$ and 4 data (2 unique) involve 4 domains, mean $I / \sigma 57.6$ The exact correlation matrix as identified by the integration program was found to be 1.00336 $0.02923-0.02720, \quad-0.01894 \quad 1.02272-0.04903,0.02520$ 0.057470 .97055 . The data were corrected for absorption using TWINABS (Sheldrick, 2009), and the structure was solved using direct methods with only the non-overlapping reflections of component 1 . The structure was refined using the HKLF5 routine with all reflections of component 1 (including the overlapping ones), resulting in a BASF value of 0.486 (1). The $R_{\text {int }}$ value given is for all reflections and is based on agreement between observed single and composite intensities and those calculated from refined unique intensities and twin fractions (TWINABS; Sheldrick, 2009). 
$\mathrm{C}$ - and O-bound $\mathrm{H}$ atoms were placed in calculated positions and allowed to ride on their carrier atoms: aromatic $\mathrm{C}-$ $\mathrm{H}_{\text {arom }}=0.95 \AA$ with $U_{\text {iso }}(\mathrm{H})=1.2 U_{\text {eq }}(\mathrm{C}), \mathrm{C}-\mathrm{H}_{\text {methyl }}=0.98 \AA$ with $U_{\text {iso }}(\mathrm{H})=1.5 U_{\text {eq }}(\mathrm{C})$. $\mathrm{O}-\mathrm{H}$ were refined for complex (I) and for hydroxide and methanol $\mathrm{H}$ atoms of complex (II), with $\mathrm{O}-\mathrm{H}$ distances restrained to 0.84 (2) $\AA$ for $\mathrm{O} 1, \mathrm{O} 3$ and $\mathrm{O} 4$ of complex (I), and $\mathrm{O} 13$ and $\mathrm{O} 14$ of complex (II) yielding $\mathrm{O}-\mathrm{H}$ distances of $0.748-0.828 \AA$. The remainder of the hydroxyl atoms were placed in calculated positions with $\mathrm{O}-\mathrm{H}=0.84 \AA$, and all $U_{\text {iso }}\left(\mathrm{H}_{\mathrm{OH}}\right)$ were set to $1.5 U_{\mathrm{eq}}(\mathrm{O})$.

\section{Acknowledgements}

The X-ray diffractometer was funded by NSF Grant 0087210 , Ohio Board of Regents Grant CAP-491, and by Youngstown State University. NSF CAREER (Grant CHE-0846383 and CHE-1360802) provided funding to ETP and her group. NSF Grant CHE-1039689 provided funding for the X-ray diffractometer at Illinois State University.

\section{References}

Addison, A. W., Rao, T. N., Reedijk, J., van Rijn, J. \& Verschoor, G. C. J. (1984). J. Chem. Soc. Dalton Trans. pp. 1349-1356.

Barnett, S. M., Goldberg, K. I. \& Mayer, J. M. (2012). Nat. Chem. 4, 498-502.

Blakemore, J. D., Schley, N. D., Balcells, D., Hull, J. F., Olack, G. W., Incarvito, C. D., Eisenstein, O., Brudvig, G. W. \& Crabtree, R. H. (2010). J. Am. Chem. Soc. 132, 16017-16029.

Bruker (2009). APEX2, SAINT and SADABS. Version 2009.7-0. Bruker AXS Inc., Madison, Wisconsin, USA.

Bruker (2012). APEX2 and SAINT. Version 2012.4-3. Bruker AXS Inc., Madison, Wisconsin, USA.

DePasquale, J., Nieto, I., Reuther, L. E., Herbst-Gervasoni, C. J., Paul, J. J., Mochalin, V., Zeller, M., Thomas, C. M., Addison, A. W. \& Papish, E. T. (2013). Inorg. Chem. 52, 9175-9183.
Gerlach, D. L., Bhagan, S., Cruce, A. A., Burks, D. B., Nieto, I., Truong, H. T., Kelley, S. P., Herbst-Gervasoni, C. J., Jernigan, K. L., Bowman, M. K., Pan, S., Zeller, M. \& Papish, E. T. (2014). Inorg. Chem. 53, 12689-12698.

Groom, C. R. \& Allen, F. H. (2014). Angew. Chem. Int. Ed. 53, 662671.

Guo, H.-X., Rao, Z.-M. \& Wang, Q.-H. (2007). Acta Cryst. E63, m637-m638.

He, X. \& Lu, C.-Z. (2004). Z. Anorg. Allg. Chem. 630, 756-759.

Hübschle, C. B., Sheldrick, G. M. \& Dittrich, B. (2011). J. Appl. Cryst. 44, 1281-1284.

Jeffrey, G. A. (2003). Crystallogr. Rev. 9, 135-176.

Kikuchi, T. \& Tanaka, K. (2014). Eur. J. Inorg. Chem. 2014, 607-618.

Luo, F., Che, Y.-X. \& Zheng, J.-M. (2006). Inorg. Chem. Commun. 9, 848-851.

Macrae, C. F., Bruno, I. J., Chisholm, J. A., Edgington, P. R., McCabe, P., Pidcock, E., Rodriguez-Monge, L., Taylor, R., van de Streek, J. \& Wood, P. A. (2008). J. Appl. Cryst. 41, 466-470.

Marelius, D. C., Bhagan, S., Charboneau, D. J., Schroeder, K. M., Kamdar, J. M., McGettigan, A. R., Freeman, B. J., Moore, C. E., Rheingold, A. L., Cooksy, A. L., Smith, D. K., Paul, J. J., Papish, E. T. \& Grotjahn, D. B. (2014). Eur. J. Inorg. Chem. pp. 676-689. Sheldrick, G. M. (2008). Acta Cryst. A64, 112-122.

Sheldrick, G. M. (2009). TWINABS. University of Göttingen, Germany.

Sheldrick, G. M. (2015). Acta Cryst. C71, 3-8.

Singh, A. \& Spiccia, L. (2013). Coord. Chem. Rev. 257, 2607-2622.

Sun, Y.-H., Yu, J.-H., Jin, X.-J., Song, J.-F., Xu, J.-Q. \& Ye, L. (2006). Inorg. Chem. Commun. 9, 1087-1090.

Wang, C.-M., Zheng, S.-T. \& Yang, G.-Y. (2009). J. Clust Sci. 20, 489 501.

Zhang, J.-P., Han, Z.-B., Chen, X.-M. \& Xuebao, W. H. (2004). Chin. J. Inorg. Chem. 20, 1213-1216.

Zhang, X.-M., Tong, M.-L. \& Chen, X.-M. (2002). Angew. Chem. Int. Ed. 41, 1029-1031.

Zhang, X.-M., Tong, M.-L., Gong, M.-L., Lee, H.-K., Luo, L., Li, K.-F., Tong, Y.-X. \& Chen, X.-M. (2002). Chem. Eur. J. 8, 3187-3194.

Zhong, Z.-G., Li, J. \& Song, J.-F. (2013). Z. Kristallogr. New Cryst. Struct. 228, 261-262.

Zhong, Z.-G., Feng, Y.-Q. \& Zhang, P. (2013). Acta Cryst. C69, 833836. 


\section{supporting information}

Acta Cryst. (2015). E71, 1447-1453 [https://doi.org/10.1107/S205698901502037X]

\section{Crystal structures of bis- and hexakis[(6,6'-dihydroxybipyridine)copper(III)] nitrate coordination complexes}

Deidra L. Gerlach, Ismael Nieto, Corey J. Herbst-Gervasoni, Gregory M. Ferrence, Matthias

Zeller and Elizabeth T. Papish

Computing details

Data collection: APEX2 (Bruker, 2009) for (I); APEX2 (Bruker, 2012) for (II). Cell refinement: SAINT (Bruker, 2009) for (I); SAINT (Bruker, 2012) for (II). Data reduction: SAINT (Bruker, 2009) for (I); SAINT (Bruker, 2012) for (II). For both compounds, program(s) used to solve structure: SHELXS97 (Sheldrick, 2008). Program(s) used to refine structure: SHELXL2014/7 (Sheldrick, 2015), SHELXLE (Hübschle et al., 2011) for (I); SHELXL2012 (Sheldrick, 2015), SHELXLE (Hübschle et al., 2011) for (II). For both compounds, molecular graphics: Mercury (Macrae et al., 2008); software used to prepare material for publication: SHELXTL (Sheldrick, 2008).

(I) $\left(6,6^{\prime}\right.$-Dihydroxybipyridine- $\left.2 \kappa^{2} N, N^{\prime}\right)\left[\mu\right.$-6-(6-hydroxypyridin-2-yl) pyridin-2-olato-1:2 $\left.\kappa^{3} N, N^{\prime}: O^{2}\right](\mu-$ hydroxido-1:2 $\left.\kappa^{2} O: O^{\prime}\right)\left(\mu\right.$-nitrato-1:2 $\left.\kappa^{2} O: O^{\prime}\right)($ nitrato-1 $\kappa O)$ dicopper(II)

\section{Crystal data}

$\left[\mathrm{Cu}_{2}\left(\mathrm{C}_{10} \mathrm{H}_{7} \mathrm{~N}_{2} \mathrm{O}_{2}\right)(\mathrm{OH})\left(\mathrm{NO}_{3}\right)_{2}\left(\mathrm{C}_{10} \mathrm{H}_{8} \mathrm{~N}_{2} \mathrm{O}_{2}\right)\right]$

$M_{r}=643.47$

Triclinic, $P \overline{1}$

$a=7.358$ (2) $\AA$

$b=10.447$ (3) $\AA$

$c=15.744$ (4) $\AA$

$\alpha=77.610(4)^{\circ}$

$\beta=78.927(4)^{\circ}$

$\gamma=69.938(4)^{\circ}$

$V=1101.1(5) \AA^{3}$

Data collection

Bruker SMART APEX CCD diffractometer

Radiation source: fine-focus sealed tube $\omega$ scans

Absorption correction: multi-scan

(SADABS; Bruker, 2009)

$T_{\min }=0.605, T_{\max }=0.746$

14887 measured reflections
$Z=2$

$F(000)=648$

$D_{\mathrm{x}}=1.941 \mathrm{Mg} \mathrm{m}^{-3}$

Mo $K \alpha$ radiation, $\lambda=0.71073 \AA$

Cell parameters from 390 reflections

$\theta=2.3-24.8^{\circ}$

$\mu=2.01 \mathrm{~mm}^{-1}$

$T=100 \mathrm{~K}$

Plate, green

$0.17 \times 0.12 \times 0.03 \mathrm{~mm}$

6413 independent reflections 4302 reflections with $I>2 \sigma(I)$

$R_{\text {int }}=0.050$

$\theta_{\text {max }}=30.7^{\circ}, \theta_{\text {min }}=1.3^{\circ}$

$h=-10 \rightarrow 10$

$k=-14 \rightarrow 14$

$l=-22 \rightarrow 21$ 


\section{Refinement}

Refinement on $F^{2}$

Least-squares matrix: full

$R\left[F^{2}>2 \sigma\left(F^{2}\right)\right]=0.052$

$w R\left(F^{2}\right)=0.125$

$S=1.04$

6413 reflections

365 parameters

3 restraints

Primary atom site location: structure-invariant direct methods
Secondary atom site location: difference Fourier map

Hydrogen site location: mixed

$\mathrm{H}$ atoms treated by a mixture of independent and constrained refinement

$w=1 /\left[\sigma^{2}\left(F_{\mathrm{o}}{ }^{2}\right)+(0.0555 P)^{2}+0.1969 P\right]$ where $P=\left(F_{\mathrm{o}}^{2}+2 F_{\mathrm{c}}^{2}\right) / 3$

$(\Delta / \sigma)_{\max }<0.001$

$\Delta \rho_{\max }=1.11 \mathrm{e} \AA^{-3}$

$\Delta \rho_{\min }=-0.69$ e $\AA^{-3}$

Special details

Geometry. All e.s.d.'s (except the e.s.d. in the dihedral angle between two 1.s. planes) are estimated using the full covariance matrix. The cell e.s.d.'s are taken into account individually in the estimation of e.s.d.'s in distances, angles and torsion angles; correlations between e.s.d.'s in cell parameters are only used when they are defined by crystal symmetry. An approximate (isotropic) treatment of cell e.s.d.'s is used for estimating e.s.d.'s involving l.s. planes.

Fractional atomic coordinates and isotropic or equivalent isotropic displacement parameters $\left(\AA^{2}\right)$

\begin{tabular}{|c|c|c|c|c|}
\hline & $x$ & $y$ & $z$ & $U_{\text {iso }} * / U_{\text {eq }}$ \\
\hline $\mathrm{C} 1$ & $0.2710(5)$ & $0.4849(4)$ & -0.0150 & $0.0185(8)$ \\
\hline $\mathrm{C} 2$ & $0.2962(6)$ & $0.5280(4)$ & -0.1049 & $0.0216(8)$ \\
\hline $\mathrm{H} 2$ & 0.2206 & 0.6168 & -0.1305 & $0.026^{*}$ \\
\hline $\mathrm{C} 3$ & $0.4322(6)$ & $0.4401(4)$ & $-0.1562(3)$ & $0.0220(8)$ \\
\hline H3 & 0.4521 & 0.4670 & -0.2180 & $0.026^{*}$ \\
\hline $\mathrm{C} 4$ & $0.5418(5)$ & $0.3099(4)$ & $-0.1166(2)$ & $0.0190(8)$ \\
\hline $\mathrm{H} 4$ & 0.6379 & 0.2480 & -0.1510 & $0.023^{*}$ \\
\hline $\mathrm{C} 5$ & $0.5083(5)$ & $0.2731(4)$ & $-0.0271(2)$ & $0.0144(7)$ \\
\hline C6 & $0.6100(5)$ & $0.1354(4)$ & $0.0196(2)$ & $0.0147(7)$ \\
\hline $\mathrm{C} 7$ & $0.7447(5)$ & 0.0339 (4) & -0.0228 & $0.0190(8)$ \\
\hline $\mathrm{H} 7$ & 0.7805 & 0.0511 & -0.0845 & $0.023^{*}$ \\
\hline $\mathrm{C} 8$ & $0.8294(5)$ & $-0.0964(4)$ & 0.0263 & $0.0187(8)$ \\
\hline H8 & 0.9244 & -0.1676 & -0.0018 & $0.022 *$ \\
\hline $\mathrm{C} 9$ & $0.7735(5)$ & $-0.1193(4)$ & $0.1146(3)$ & $0.0174(8)$ \\
\hline H9 & 0.8290 & -0.2065 & 0.1486 & $0.021 *$ \\
\hline $\mathrm{C} 10$ & $0.6315(5)$ & $-0.0113(4)$ & $0.1552(2)$ & $0.0140(7)$ \\
\hline C11 & $0.2728(5)$ & $0.2782(4)$ & $0.4525(2)$ & $0.0169(7)$ \\
\hline $\mathrm{C} 12$ & $0.1854(5)$ & $0.3244(4)$ & 0.5324 & $0.0186(8)$ \\
\hline H12 & 0.1660 & 0.4165 & 0.5392 & $0.022 *$ \\
\hline C13 & $0.1285(5)$ & $0.2346(4)$ & 0.6005 & $0.0201(8)$ \\
\hline H13 & 0.0705 & 0.2638 & 0.6553 & $0.024 *$ \\
\hline $\mathrm{C} 14$ & $0.1560(5)$ & 0.0991 (4) & $0.5894(2)$ & $0.0175(7)$ \\
\hline H14 & 0.1177 & 0.0355 & 0.6362 & $0.021 *$ \\
\hline $\mathrm{C} 15$ & $0.2398(5)$ & $0.0608(4)$ & $0.5090(2)$ & $0.0138(7)$ \\
\hline H15 & $0.584(6)$ & $0.211(4)$ & $0.257(3)$ & $0.016(12)^{*}$ \\
\hline $\mathrm{C} 16$ & $0.2731(5)$ & $-0.0791(4)$ & $0.4908(2)$ & $0.0147(7)$ \\
\hline $\mathrm{C} 17$ & $0.2193(5)$ & -0.1803 & $0.5517(2)$ & $0.0164(7)$ \\
\hline H17 & 0.1568 & -0.1622 & 0.6085 & $0.020 *$ \\
\hline
\end{tabular}




$\begin{array}{lllll}\text { C18 } & 0.2577(5) & -0.3093(4) & 0.5287(2) & 0.0172(7) \\ \text { H18 } & 0.2232 & -0.3806 & 0.5702 & 0.021^{*} \\ \text { C19 } & 0.3449(5) & -0.3331(4) & 0.4465(3) & 0.0191(8) \\ \text { H19 } & 0.3704 & -0.4204 & 0.4299 & 0.023^{*} \\ \text { C20 } & 0.3961(5) & -0.2269(4) & 0.3869(2) & 0.0172(7) \\ \text { N1 } & 0.3732(4) & 0.3613(3) & 0.0244(2) & 0.0156(6) \\ \text { N2 } & 0.5553(4) & 0.1137(3) & 0.10808(19) & 0.0129(6) \\ \text { N3 } & 0.2987(4) & 0.1489(3) & 0.44065(19) & 0.0126(6) \\ \text { N4 } & 0.3616(4) & -0.1019(3) & 0.40843(19) & 0.0139(6) \\ \text { N5 } & 0.0443(5) & 0.4545(4) & 0.2519(2) & 0.0217(7) \\ \text { N6 } & 0.0449(4) & 0.1339(3) & 0.2131(2) & 0.0149(6) \\ \text { O1 } & 0.1390(4) & 0.5753(3) & 0.03228(18) & 0.0244(6) \\ \text { H1 } & 0.140(7) & 0.552(5) & 0.0859(14) & 0.037^{*} \\ \text { O2 } & 0.5740(4) & -0.0383(3) & 0.23919(17) & 0.0181(5) \\ \text { O3 } & 0.3321(4) & 0.3654(3) & 0.38846(17) & 0.0194(6) \\ \text { H3B } & 0.380(6) & 0.334(5) & 0.3421(19) & 0.029^{*} \\ \text { O4 } & 0.4801(4) & -0.2525(3) & 0.30803(18) & 0.0217(6) \\ \text { H4B } & 0.532(6) & -0.195(4) & 0.280(3) & 0.033^{*} \\ \text { O5 } & 0.4755(4) & 0.2277(3) & 0.26553(17) & 0.0159(5) \\ \text { O6 } & 0.1872(4) & 0.4522(3) & 0.19101(18) & 0.0208(6) \\ \text { O7 } & 0.0328(4) & 0.3455(3) & 0.29893(19) & 0.0294(7) \\ \text { O8 } & -0.0797(4) & 0.5672(3) & 0.2623(2) & 0.0367(8) \\ \text { O9 } & 0.1131(4) & 0.2090(3) & 0.15141(17) & 0.0205(6) \\ \text { O10 } & 0.1392(4) & 0.0637(3) & 0.2725(2) & 0.0290(7) \\ \text { O11 } & -0.1223(4) & 0.1285(3) & 0.21196(19) & 0.0260(7) \\ \text { Cu1 } & 0.36658(6) & 0.27682(5) & 0.15407(3) & 0.01478(12) \\ \text { Cu2 } & 0.41333(6) & 0.06500(5) & 0.33097(3) & 0.01386(12) \\ & & & & \end{array}$

Atomic displacement parameters $\left(\AA^{2}\right)$

\begin{tabular}{lllllll}
\hline & $U^{11}$ & $U^{22}$ & $U^{33}$ & $U^{12}$ & $U^{13}$ & $U^{23}$ \\
\hline C1 & $0.0167(17)$ & $0.0172(19)$ & $0.024(2)$ & $-0.0082(15)$ & $-0.0056(15)$ & $-0.0011(16)$ \\
C2 & $0.0243(19)$ & $0.0138(19)$ & $0.027(2)$ & $-0.0079(15)$ & $-0.0079(16)$ & $0.0036(16)$ \\
C3 & $0.027(2)$ & $0.023(2)$ & $0.0168(19)$ & $-0.0125(17)$ & $-0.0023(15)$ & $0.0030(16)$ \\
C4 & $0.0224(19)$ & $0.020(2)$ & $0.0154(18)$ & $-0.0094(16)$ & $-0.0001(15)$ & $-0.0017(15)$ \\
C5 & $0.0112(15)$ & $0.0162(19)$ & $0.0183(18)$ & $-0.0070(14)$ & $-0.0005(13)$ & $-0.0045(15)$ \\
C6 & $0.0107(15)$ & $0.0180(19)$ & $0.0172(18)$ & $-0.0076(14)$ & $-0.0024(13)$ & $-0.0010(15)$ \\
C7 & $0.0167(17)$ & $0.020(2)$ & $0.0198(19)$ & $-0.0075(15)$ & $0.0012(14)$ & $-0.0027(16)$ \\
C8 & $0.0131(16)$ & $0.0170(19)$ & $0.025(2)$ & $-0.0026(14)$ & $-0.0009(14)$ & $-0.0059(16)$ \\
C9 & $0.0140(16)$ & $0.0138(18)$ & $0.025(2)$ & $-0.0032(14)$ & $-0.0032(14)$ & $-0.0045(15)$ \\
C10 & $0.0134(16)$ & $0.0148(18)$ & $0.0160(18)$ & $-0.0070(14)$ & $0.0003(13)$ & $-0.0045(14)$ \\
C11 & $0.0169(17)$ & $0.0137(18)$ & $0.0212(19)$ & $-0.0045(14)$ & $-0.0071(14)$ & $-0.0013(15)$ \\
C12 & $0.0181(17)$ & $0.0148(19)$ & $0.024(2)$ & $-0.0034(14)$ & $-0.0059(15)$ & $-0.0064(16)$ \\
C13 & $0.0196(18)$ & $0.024(2)$ & $0.0180(19)$ & $-0.0063(16)$ & $-0.0011(14)$ & $-0.0077(16)$ \\
C14 & $0.0155(17)$ & $0.019(2)$ & $0.0184(18)$ & $-0.0062(15)$ & $-0.0027(14)$ & $-0.0034(15)$ \\
C15 & $0.0117(15)$ & $0.0145(18)$ & $0.0155(17)$ & $-0.0044(13)$ & $-0.0026(13)$ & $-0.0015(14)$ \\
C16 & $0.0110(15)$ & $0.0150(18)$ & $0.0178(18)$ & $-0.0027(13)$ & $-0.0032(13)$ & $-0.0028(14)$ \\
C17 & $0.0165(17)$ & $0.0191(19)$ & $0.0142(17)$ & $-0.0083(15)$ & $-0.0011(13)$ & $-0.0003(15)$
\end{tabular}




$\begin{array}{lllllll}\text { C18 } & 0.0154(17) & 0.0164(19) & 0.0203(19) & -0.0080(14) & -0.0030(14) & 0.0014(15) \\ \text { C19 } & 0.0198(18) & 0.0096(18) & 0.028(2) & -0.0055(14) & -0.0022(15) & -0.0036(15) \\ \text { C20 } & 0.0170(17) & 0.0154(19) & 0.0189(19) & -0.0037(14) & -0.0045(14) & -0.0027(15) \\ \text { N1 } & 0.0141(14) & 0.0139(15) & 0.0193(16) & -0.0065(12) & -0.0032(12) & 0.0006(13) \\ \text { N2 } & 0.0117(13) & 0.0128(15) & 0.0139(15) & -0.0040(11) & -0.0006(11) & -0.0025(12) \\ \text { N3 } & 0.0120(13) & 0.0114(15) & 0.0143(14) & -0.0028(11) & -0.0021(11) & -0.0030(12) \\ \text { N4 } & 0.0126(14) & 0.0133(15) & 0.0156(15) & -0.0035(11) & -0.0029(11) & -0.0020(12) \\ \text { N5 } & 0.0220(16) & 0.0194(18) & 0.0257(18) & -0.0022(14) & -0.0097(14) & -0.0092(15) \\ \text { N6 } & 0.0140(14) & 0.0127(15) & 0.0178(16) & -0.0039(12) & 0.0004(12) & -0.0050(13) \\ \text { O1 } & 0.0309(15) & 0.0146(14) & 0.0209(15) & 0.0007(12) & -0.0024(12) & -0.0024(12) \\ \text { O2 } & 0.0208(13) & 0.0147(13) & 0.0151(13) & -0.0051(10) & 0.0007(10) & 0.0016(11) \\ \text { O3 } & 0.0295(15) & 0.0160(14) & 0.0154(13) & -0.0103(11) & -0.0021(11) & -0.0036(11) \\ \text { O4 } & 0.0325(15) & 0.0169(14) & 0.0171(14) & -0.0110(12) & 0.0049(11) & -0.0076(11) \\ \text { O5 } & 0.0151(13) & 0.0151(13) & 0.0186(14) & -0.0067(11) & -0.0021(10) & -0.0019(11) \\ \text { O6 } & 0.0240(14) & 0.0142(14) & 0.0225(14) & -0.0041(11) & -0.0024(11) & -0.0027(11) \\ \text { O7 } & 0.0319(16) & 0.0290(17) & 0.0274(16) & -0.0131(13) & 0.0039(13) & -0.0061(14) \\ \text { O8 } & 0.0320(17) & 0.0305(18) & 0.0410(19) & 0.0030(14) & 0.0008(14) & -0.0180(15) \\ \text { O9 } & 0.0215(13) & 0.0256(15) & 0.0160(13) & -0.0125(12) & 0.0007(10) & -0.0013(12) \\ \text { O10 } & 0.0244(15) & 0.0310(17) & 0.0330(17) & -0.0126(13) & -0.0151(13) & 0.0083(14) \\ \text { O11 } & 0.0140(13) & 0.0296(16) & 0.0354(17) & -0.0104(12) & -0.0033(12) & -0.0012(14) \\ \text { Cu1 } & 0.0146(2) & 0.0141(2) & 0.0145(2) & -0.00367(17) & -0.00074(16) & -0.00225(18) \\ \text { Cu2 } & 0.0155(2) & 0.0128(2) & 0.0137(2) & -0.00583(17) & -0.00062(16) & -0.00191(17)\end{array}$

Geometric parameters ( $\left.\AA,{ }^{\circ}\right)$

\begin{tabular}{|c|c|c|c|}
\hline $\mathrm{C} 1-\mathrm{O} 1$ & $1.335(5)$ & $\mathrm{C} 15-\mathrm{C} 16$ & $1.478(5)$ \\
\hline $\mathrm{C} 1-\mathrm{N} 1$ & $1.336(5)$ & $\mathrm{C} 16-\mathrm{N} 4$ & $1.366(4)$ \\
\hline $\mathrm{C} 1-\mathrm{C} 2$ & $1.388(6)$ & $\mathrm{C} 16-\mathrm{C} 17$ & $1.378(5)$ \\
\hline $\mathrm{C} 2-\mathrm{C} 3$ & $1.369(6)$ & $\mathrm{C} 17-\mathrm{C} 18$ & $1.391(5)$ \\
\hline $\mathrm{C} 2-\mathrm{H} 2$ & 0.9500 & $\mathrm{C} 17-\mathrm{H} 17$ & 0.9500 \\
\hline $\mathrm{C} 3-\mathrm{C} 4$ & $1.402(5)$ & $\mathrm{C} 18-\mathrm{C} 19$ & $1.363(5)$ \\
\hline $\mathrm{C} 3-\mathrm{H} 3$ & 0.9500 & $\mathrm{C} 18-\mathrm{H} 18$ & 0.9500 \\
\hline $\mathrm{C} 4-\mathrm{C} 5$ & $1.376(5)$ & $\mathrm{C} 19-\mathrm{C} 20$ & $1.399(5)$ \\
\hline $\mathrm{C} 4-\mathrm{H} 4$ & 0.9500 & C19-H19 & 0.9500 \\
\hline $\mathrm{C} 5-\mathrm{N} 1$ & $1.370(4)$ & $\mathrm{C} 20-\mathrm{O} 4$ & $1.316(4)$ \\
\hline $\mathrm{C} 5-\mathrm{C} 6$ & $1.480(5)$ & $\mathrm{C} 20-\mathrm{N} 4$ & $1.346(5)$ \\
\hline $\mathrm{C} 6-\mathrm{N} 2$ & $1.366(5)$ & $\mathrm{N} 1-\mathrm{Cu} 1$ & $2.042(3)$ \\
\hline $\mathrm{C} 6-\mathrm{C} 7$ & $1.372(5)$ & $\mathrm{N} 2-\mathrm{Cu} 1$ & $1.969(3)$ \\
\hline $\mathrm{C} 7-\mathrm{C} 8$ & $1.411(5)$ & $\mathrm{N} 3-\mathrm{Cu} 2$ & $2.011(3)$ \\
\hline $\mathrm{C} 7-\mathrm{H} 7$ & 0.9500 & $\mathrm{~N} 4-\mathrm{Cu} 2$ & 2.009 (3) \\
\hline $\mathrm{C} 8-\mathrm{C} 9$ & $1.366(5)$ & $\mathrm{N} 5-\mathrm{O} 8$ & $1.238(4)$ \\
\hline $\mathrm{C} 8-\mathrm{H} 8$ & 0.9500 & $\mathrm{~N} 5-\mathrm{O} 7$ & $1.239(4)$ \\
\hline $\mathrm{C} 9-\mathrm{C} 10$ & $1.420(5)$ & N5-O6 & $1.276(4)$ \\
\hline C9-H9 & 0.9500 & $\mathrm{~N} 6-\mathrm{O} 10$ & $1.225(4)$ \\
\hline $\mathrm{C} 10-\mathrm{O} 2$ & $1.310(4)$ & N6-O9 & $1.251(4)$ \\
\hline $\mathrm{C} 10-\mathrm{N} 2$ & $1.346(5)$ & N6-O11 & $1.254(4)$ \\
\hline $\mathrm{C} 11-\mathrm{O} 3$ & $1.322(5)$ & $\mathrm{O} 1-\mathrm{H} 1$ & $0.827(19)$ \\
\hline $\mathrm{C} 11-\mathrm{N} 3$ & $1.347(5)$ & $\mathrm{O} 2-\mathrm{Cu} 2$ & $1.946(3)$ \\
\hline
\end{tabular}




\begin{tabular}{|c|c|c|c|}
\hline $\mathrm{C} 11-\mathrm{C} 12$ & $1.402(5)$ & $\mathrm{O} 3-\mathrm{H} 3 \mathrm{~B}$ & $0.829(19)$ \\
\hline $\mathrm{C} 12-\mathrm{C} 13$ & $1.367(5)$ & $\mathrm{O} 4-\mathrm{H} 4 \mathrm{~B}$ & $0.827(19)$ \\
\hline $\mathrm{C} 12-\mathrm{H} 12$ & 0.9500 & $\mathrm{O} 5-\mathrm{Cu} 2$ & $1.939(3)$ \\
\hline $\mathrm{C} 13-\mathrm{C} 14$ & $1.404(5)$ & $\mathrm{O} 5-\mathrm{Cu} 1$ & $1.964(3)$ \\
\hline $\mathrm{C} 13-\mathrm{H} 13$ & 0.9500 & $\mathrm{O} 5-\mathrm{H} 15$ & $0.75(4)$ \\
\hline $\mathrm{C} 14-\mathrm{C} 15$ & $1.374(5)$ & $\mathrm{O} 6-\mathrm{Cu} 1$ & $1.985(3)$ \\
\hline $\mathrm{C} 14-\mathrm{H} 14$ & 0.9500 & $\mathrm{O} 9-\mathrm{Cu} 1$ & $2.221(3)$ \\
\hline $\mathrm{C} 15-\mathrm{N} 3$ & $1.365(5)$ & $\mathrm{O} 10-\mathrm{Cu} 2$ & $2.377(3)$ \\
\hline $\mathrm{O} 1-\mathrm{C} 1-\mathrm{N} 1$ & $120.4(3)$ & $\mathrm{C} 18-\mathrm{C} 19-\mathrm{C} 20$ & $119.0(4)$ \\
\hline $\mathrm{O} 1-\mathrm{C} 1-\mathrm{C} 2$ & $116.4(3)$ & $\mathrm{C} 18-\mathrm{C} 19-\mathrm{H} 19$ & 120.5 \\
\hline $\mathrm{N} 1-\mathrm{C} 1-\mathrm{C} 2$ & $123.2(4)$ & $\mathrm{C} 20-\mathrm{C} 19-\mathrm{H} 19$ & 120.5 \\
\hline $\mathrm{C} 3-\mathrm{C} 2-\mathrm{C} 1$ & $118.7(4)$ & $\mathrm{O} 4-\mathrm{C} 20-\mathrm{N} 4$ & $120.3(3)$ \\
\hline $\mathrm{C} 3-\mathrm{C} 2-\mathrm{H} 2$ & 120.7 & $\mathrm{O} 4-\mathrm{C} 20-\mathrm{C} 19$ & $117.8(3)$ \\
\hline $\mathrm{C} 1-\mathrm{C} 2-\mathrm{H} 2$ & 120.7 & $\mathrm{~N} 4-\mathrm{C} 20-\mathrm{C} 19$ & $121.9(3)$ \\
\hline $\mathrm{C} 2-\mathrm{C} 3-\mathrm{C} 4$ & $119.3(4)$ & $\mathrm{C} 1-\mathrm{N} 1-\mathrm{C} 5$ & $118.0(3)$ \\
\hline $\mathrm{C} 2-\mathrm{C} 3-\mathrm{H} 3$ & 120.3 & $\mathrm{C} 1-\mathrm{N} 1-\mathrm{Cu} 1$ & $130.4(3)$ \\
\hline $\mathrm{C} 4-\mathrm{C} 3-\mathrm{H} 3$ & 120.3 & $\mathrm{C} 5-\mathrm{N} 1-\mathrm{Cu} 1$ & $111.6(2)$ \\
\hline $\mathrm{C} 5-\mathrm{C} 4-\mathrm{C} 3$ & $119.1(4)$ & $\mathrm{C} 10-\mathrm{N} 2-\mathrm{C} 6$ & $119.6(3)$ \\
\hline $\mathrm{C} 5-\mathrm{C} 4-\mathrm{H} 4$ & 120.5 & $\mathrm{C} 10-\mathrm{N} 2-\mathrm{Cu} 1$ & $126.3(2)$ \\
\hline $\mathrm{C} 3-\mathrm{C} 4-\mathrm{H} 4$ & 120.5 & $\mathrm{C} 6-\mathrm{N} 2-\mathrm{Cu} 1$ & $114.1(2)$ \\
\hline $\mathrm{N} 1-\mathrm{C} 5-\mathrm{C} 4$ & $121.7(3)$ & $\mathrm{C} 11-\mathrm{N} 3-\mathrm{C} 15$ & $118.6(3)$ \\
\hline $\mathrm{N} 1-\mathrm{C} 5-\mathrm{C} 6$ & $115.6(3)$ & $\mathrm{C} 11-\mathrm{N} 3-\mathrm{Cu} 2$ & $127.8(2)$ \\
\hline $\mathrm{C} 4-\mathrm{C} 5-\mathrm{C} 6$ & $122.7(3)$ & $\mathrm{C} 15-\mathrm{N} 3-\mathrm{Cu} 2$ & $113.6(2)$ \\
\hline $\mathrm{N} 2-\mathrm{C} 6-\mathrm{C} 7$ & $121.8(3)$ & $\mathrm{C} 20-\mathrm{N} 4-\mathrm{C} 16$ & $118.6(3)$ \\
\hline $\mathrm{N} 2-\mathrm{C} 6-\mathrm{C} 5$ & $115.4(3)$ & $\mathrm{C} 20-\mathrm{N} 4-\mathrm{Cu} 2$ & $127.7(2)$ \\
\hline $\mathrm{C} 7-\mathrm{C} 6-\mathrm{C} 5$ & $122.7(3)$ & $\mathrm{C} 16-\mathrm{N} 4-\mathrm{Cu} 2$ & $113.7(2)$ \\
\hline $\mathrm{C} 6-\mathrm{C} 7-\mathrm{C} 8$ & $119.1(3)$ & $\mathrm{O} 8-\mathrm{N} 5-\mathrm{O} 7$ & $121.6(4)$ \\
\hline $\mathrm{C} 6-\mathrm{C} 7-\mathrm{H} 7$ & 120.4 & $\mathrm{O} 8-\mathrm{N} 5-\mathrm{O} 6$ & $118.6(4)$ \\
\hline $\mathrm{C} 8-\mathrm{C} 7-\mathrm{H} 7$ & 120.4 & $\mathrm{O} 7-\mathrm{N} 5-\mathrm{O} 6$ & $119.8(3)$ \\
\hline $\mathrm{C} 9-\mathrm{C} 8-\mathrm{C} 7$ & $119.4(3)$ & $\mathrm{O} 10-\mathrm{N} 6-\mathrm{O} 9$ & $121.6(3)$ \\
\hline $\mathrm{C} 9-\mathrm{C} 8-\mathrm{H} 8$ & 120.3 & $\mathrm{O} 10-\mathrm{N} 6-\mathrm{O} 11$ & $119.9(3)$ \\
\hline $\mathrm{C} 7-\mathrm{C} 8-\mathrm{H} 8$ & 120.3 & $\mathrm{O} 9-\mathrm{N} 6-\mathrm{O} 11$ & $118.5(3)$ \\
\hline $\mathrm{C} 8-\mathrm{C} 9-\mathrm{C} 10$ & $119.3(4)$ & $\mathrm{C} 1-\mathrm{O} 1-\mathrm{H} 1$ & $114(3)$ \\
\hline $\mathrm{C} 8-\mathrm{C} 9-\mathrm{H} 9$ & 120.4 & $\mathrm{C} 10-\mathrm{O} 2-\mathrm{Cu} 2$ & $137.1(2)$ \\
\hline $\mathrm{C} 10-\mathrm{C} 9-\mathrm{H} 9$ & 120.4 & $\mathrm{C} 11-\mathrm{O} 3-\mathrm{H} 3 \mathrm{~B}$ & $114(3)$ \\
\hline $\mathrm{O} 2-\mathrm{C} 10-\mathrm{N} 2$ & $121.2(3)$ & $\mathrm{C} 20-\mathrm{O} 4-\mathrm{H} 4 \mathrm{~B}$ & $114(3)$ \\
\hline $\mathrm{O} 2-\mathrm{C} 10-\mathrm{C} 9$ & $117.9(3)$ & $\mathrm{Cu} 2-\mathrm{O} 5-\mathrm{Cu} 1$ & $108.02(13)$ \\
\hline $\mathrm{N} 2-\mathrm{C} 10-\mathrm{C} 9$ & $120.9(3)$ & $\mathrm{Cu} 2-\mathrm{O} 5-\mathrm{H} 15$ & $108(3)$ \\
\hline $\mathrm{O} 3-\mathrm{C} 11-\mathrm{N} 3$ & $120.3(3)$ & $\mathrm{Cu} 1-\mathrm{O} 5-\mathrm{H} 15$ & $110(3)$ \\
\hline $\mathrm{O} 3-\mathrm{C} 11-\mathrm{C} 12$ & $117.9(3)$ & $\mathrm{N} 5-\mathrm{O} 6-\mathrm{Cu} 1$ & $121.9(2)$ \\
\hline $\mathrm{N} 3-\mathrm{C} 11-\mathrm{C} 12$ & $121.8(3)$ & $\mathrm{N} 6-\mathrm{O} 9-\mathrm{Cu} 1$ & $124.9(2)$ \\
\hline $\mathrm{C} 13-\mathrm{C} 12-\mathrm{C} 11$ & $118.9(4)$ & $\mathrm{N} 6-\mathrm{O} 10-\mathrm{Cu} 2$ & $135.9(2)$ \\
\hline $\mathrm{C} 13-\mathrm{C} 12-\mathrm{H} 12$ & 120.5 & $\mathrm{O} 5-\mathrm{Cu} 1-\mathrm{N} 2$ & $92.97(12)$ \\
\hline $\mathrm{C} 11-\mathrm{C} 12-\mathrm{H} 12$ & 120.5 & $\mathrm{O} 5-\mathrm{Cu} 1-\mathrm{O} 6$ & 89.59 (11) \\
\hline $\mathrm{C} 12-\mathrm{C} 13-\mathrm{C} 14$ & $119.9(4)$ & $\mathrm{N} 2-\mathrm{Cu} 1-\mathrm{O} 6$ & $174.53(12)$ \\
\hline $\mathrm{C} 12-\mathrm{C} 13-\mathrm{H} 13$ & 120.0 & $\mathrm{O} 5-\mathrm{Cu} 1-\mathrm{N} 1$ & $150.90(12)$ \\
\hline $\mathrm{C} 14-\mathrm{C} 13-\mathrm{H} 13$ & 120.0 & $\mathrm{~N} 2-\mathrm{Cu} 1-\mathrm{N} 1$ & $82.98(12)$ \\
\hline
\end{tabular}




\begin{tabular}{|c|c|c|c|}
\hline $\mathrm{C} 15-\mathrm{C} 14-\mathrm{C} 13$ & $118.3(4)$ & $\mathrm{O} 6-\mathrm{Cu} 1-\mathrm{N} 1$ & $92.44(12)$ \\
\hline $\mathrm{C} 15-\mathrm{C} 14-\mathrm{H} 14$ & 120.8 & $\mathrm{O} 5-\mathrm{Cu} 1-\mathrm{O} 9$ & $116.83(10)$ \\
\hline $\mathrm{C} 13-\mathrm{C} 14-\mathrm{H} 14$ & 120.8 & $\mathrm{~N} 2-\mathrm{Cu} 1-\mathrm{O} 9$ & $92.96(11)$ \\
\hline $\mathrm{N} 3-\mathrm{C} 15-\mathrm{C} 14$ & $122.4(3)$ & $\mathrm{O} 6-\mathrm{Cu} 1-\mathrm{O} 9$ & $90.19(11)$ \\
\hline $\mathrm{N} 3-\mathrm{C} 15-\mathrm{C} 16$ & $115.4(3)$ & $\mathrm{N} 1-\mathrm{Cu} 1-\mathrm{O} 9$ & $92.20(11)$ \\
\hline $\mathrm{C} 14-\mathrm{C} 15-\mathrm{C} 16$ & $122.2(3)$ & $\mathrm{O} 5-\mathrm{Cu} 2-\mathrm{O} 2$ & $89.00(11)$ \\
\hline $\mathrm{N} 4-\mathrm{C} 16-\mathrm{C} 17$ & $121.7(3)$ & $\mathrm{O} 5-\mathrm{Cu} 2-\mathrm{N} 4$ & $174.31(12)$ \\
\hline $\mathrm{N} 4-\mathrm{C} 16-\mathrm{C} 15$ & $115.1(3)$ & $\mathrm{O} 2-\mathrm{Cu} 2-\mathrm{N} 4$ & $93.75(11)$ \\
\hline $\mathrm{C} 17-\mathrm{C} 16-\mathrm{C} 15$ & $123.2(3)$ & $\mathrm{O} 5-\mathrm{Cu} 2-\mathrm{N} 3$ & $94.05(12)$ \\
\hline $\mathrm{C} 16-\mathrm{C} 17-\mathrm{C} 18$ & $119.0(3)$ & $\mathrm{O} 2-\mathrm{Cu} 2-\mathrm{N} 3$ & $167.14(11)$ \\
\hline $\mathrm{C} 16-\mathrm{C} 17-\mathrm{H} 17$ & 120.5 & $\mathrm{~N} 4-\mathrm{Cu} 2-\mathrm{N} 3$ & $82.20(12)$ \\
\hline $\mathrm{C} 18-\mathrm{C} 17-\mathrm{H} 17$ & 120.5 & $\mathrm{O} 5-\mathrm{Cu} 2-\mathrm{O} 10$ & $104.96(11)$ \\
\hline $\mathrm{C} 19-\mathrm{C} 18-\mathrm{C} 17$ & $119.8(3)$ & $\mathrm{O} 2-\mathrm{Cu} 2-\mathrm{O} 10$ & $86.67(11)$ \\
\hline $\mathrm{C} 19-\mathrm{C} 18-\mathrm{H} 18$ & 120.1 & $\mathrm{~N} 4-\mathrm{Cu} 2-\mathrm{O} 10$ & $80.20(11)$ \\
\hline $\mathrm{C} 17-\mathrm{C} 18-\mathrm{H} 18$ & 120.1 & $\mathrm{~N} 3-\mathrm{Cu} 2-\mathrm{O} 10$ & $104.54(11)$ \\
\hline $\mathrm{O} 1-\mathrm{C} 1-\mathrm{C} 2-\mathrm{C} 3$ & $178.8(3)$ & $\mathrm{C} 4-\mathrm{C} 5-\mathrm{N} 1-\mathrm{C} 1$ & $1.1(5)$ \\
\hline $\mathrm{N} 1-\mathrm{C} 1-\mathrm{C} 2-\mathrm{C} 3$ & $0.0(6)$ & $\mathrm{C} 6-\mathrm{C} 5-\mathrm{N} 1-\mathrm{C} 1$ & $-177.1(3)$ \\
\hline $\mathrm{C} 1-\mathrm{C} 2-\mathrm{C} 3-\mathrm{C} 4$ & $-0.2(6)$ & $\mathrm{C} 4-\mathrm{C} 5-\mathrm{N} 1-\mathrm{Cu} 1$ & $-178.2(3)$ \\
\hline $\mathrm{C} 2-\mathrm{C} 3-\mathrm{C} 4-\mathrm{C} 5$ & $0.7(6)$ & $\mathrm{C} 6-\mathrm{C} 5-\mathrm{N} 1-\mathrm{Cu} 1$ & $3.6(4)$ \\
\hline $\mathrm{C} 3-\mathrm{C} 4-\mathrm{C} 5-\mathrm{N} 1$ & $-1.2(5)$ & $\mathrm{O} 2-\mathrm{C} 10-\mathrm{N} 2-\mathrm{C} 6$ & $-176.0(3)$ \\
\hline $\mathrm{C} 3-\mathrm{C} 4-\mathrm{C} 5-\mathrm{C} 6$ & $176.9(3)$ & $\mathrm{C} 9-\mathrm{C} 10-\mathrm{N} 2-\mathrm{C} 6$ & $2.4(5)$ \\
\hline $\mathrm{N} 1-\mathrm{C} 5-\mathrm{C} 6-\mathrm{N} 2$ & $0.3(5)$ & $\mathrm{O} 2-\mathrm{C} 10-\mathrm{N} 2-\mathrm{Cu} 1$ & $4.4(5)$ \\
\hline $\mathrm{C} 4-\mathrm{C} 5-\mathrm{C} 6-\mathrm{N} 2$ & $-177.8(3)$ & $\mathrm{C} 9-\mathrm{C} 10-\mathrm{N} 2-\mathrm{Cu} 1$ & $-177.2(3)$ \\
\hline $\mathrm{N} 1-\mathrm{C} 5-\mathrm{C} 6-\mathrm{C} 7$ & $177.9(3)$ & $\mathrm{C} 7-\mathrm{C} 6-\mathrm{N} 2-\mathrm{C} 10$ & $-1.5(5)$ \\
\hline $\mathrm{C} 4-\mathrm{C} 5-\mathrm{C} 6-\mathrm{C} 7$ & $-0.3(6)$ & $\mathrm{C} 5-\mathrm{C} 6-\mathrm{N} 2-\mathrm{C} 10$ & $176.0(3)$ \\
\hline $\mathrm{N} 2-\mathrm{C} 6-\mathrm{C} 7-\mathrm{C} 8$ & $-0.2(5)$ & $\mathrm{C} 7-\mathrm{C} 6-\mathrm{N} 2-\mathrm{Cu} 1$ & $178.1(3)$ \\
\hline $\mathrm{C} 5-\mathrm{C} 6-\mathrm{C} 7-\mathrm{C} 8$ & $-177.5(3)$ & $\mathrm{C} 5-\mathrm{C} 6-\mathrm{N} 2-\mathrm{Cu} 1$ & $-4.3(4)$ \\
\hline $\mathrm{C} 6-\mathrm{C} 7-\mathrm{C} 8-\mathrm{C} 9$ & $1.0(5)$ & $\mathrm{O} 3-\mathrm{C} 11-\mathrm{N} 3-\mathrm{C} 15$ & $178.4(3)$ \\
\hline $\mathrm{C} 7-\mathrm{C} 8-\mathrm{C} 9-\mathrm{C} 10$ & $-0.1(5)$ & $\mathrm{C} 12-\mathrm{C} 11-\mathrm{N} 3-\mathrm{C} 15$ & $-0.8(5)$ \\
\hline $\mathrm{C} 8-\mathrm{C} 9-\mathrm{C} 10-\mathrm{O} 2$ & $176.8(3)$ & $\mathrm{O} 3-\mathrm{C} 11-\mathrm{N} 3-\mathrm{Cu} 2$ & $-2.6(5)$ \\
\hline $\mathrm{C} 8-\mathrm{C} 9-\mathrm{C} 10-\mathrm{N} 2$ & $-1.6(5)$ & $\mathrm{C} 12-\mathrm{C} 11-\mathrm{N} 3-\mathrm{Cu} 2$ & $178.2(3)$ \\
\hline $\mathrm{O} 3-\mathrm{C} 11-\mathrm{C} 12-\mathrm{C} 13$ & $-177.9(3)$ & $\mathrm{C} 14-\mathrm{C} 15-\mathrm{N} 3-\mathrm{C} 11$ & $-0.3(5)$ \\
\hline $\mathrm{N} 3-\mathrm{C} 11-\mathrm{C} 12-\mathrm{C} 13$ & $1.3(5)$ & $\mathrm{C} 16-\mathrm{C} 15-\mathrm{N} 3-\mathrm{C} 11$ & $180.0(3)$ \\
\hline $\mathrm{C} 11-\mathrm{C} 12-\mathrm{C} 13-\mathrm{C} 14$ & $-0.7(6)$ & $\mathrm{C} 14-\mathrm{C} 15-\mathrm{N} 3-\mathrm{Cu} 2$ & $-179.4(3)$ \\
\hline $\mathrm{C} 12-\mathrm{C} 13-\mathrm{C} 14-\mathrm{C} 15$ & $-0.3(5)$ & $\mathrm{C} 16-\mathrm{C} 15-\mathrm{N} 3-\mathrm{Cu} 2$ & $0.9(4)$ \\
\hline $\mathrm{C} 13-\mathrm{C} 14-\mathrm{C} 15-\mathrm{N} 3$ & $0.8(5)$ & $\mathrm{O} 4-\mathrm{C} 20-\mathrm{N} 4-\mathrm{C} 16$ & $179.6(3)$ \\
\hline $\mathrm{C} 13-\mathrm{C} 14-\mathrm{C} 15-\mathrm{C} 16$ & $-179.5(3)$ & $\mathrm{C} 19-\mathrm{C} 20-\mathrm{N} 4-\mathrm{C} 16$ & $-0.3(5)$ \\
\hline $\mathrm{N} 3-\mathrm{C} 15-\mathrm{C} 16-\mathrm{N} 4$ & $1.1(4)$ & $\mathrm{O} 4-\mathrm{C} 20-\mathrm{N} 4-\mathrm{Cu} 2$ & $2.8(5)$ \\
\hline $\mathrm{C} 14-\mathrm{C} 15-\mathrm{C} 16-\mathrm{N} 4$ & $-178.6(3)$ & $\mathrm{C} 19-\mathrm{C} 20-\mathrm{N} 4-\mathrm{Cu} 2$ & $-177.1(3)$ \\
\hline $\mathrm{N} 3-\mathrm{C} 15-\mathrm{C} 16-\mathrm{C} 17$ & $-178.7(3)$ & $\mathrm{C} 17-\mathrm{C} 16-\mathrm{N} 4-\mathrm{C} 20$ & $0.0(5)$ \\
\hline $\mathrm{C} 14-\mathrm{C} 15-\mathrm{C} 16-\mathrm{C} 17$ & $1.6(5)$ & $\mathrm{C} 15-\mathrm{C} 16-\mathrm{N} 4-\mathrm{C} 20$ & $-179.8(3)$ \\
\hline $\mathrm{N} 4-\mathrm{C} 16-\mathrm{C} 17-\mathrm{C} 18$ & $0.6(5)$ & $\mathrm{C} 17-\mathrm{C} 16-\mathrm{N} 4-\mathrm{Cu} 2$ & $177.3(3)$ \\
\hline $\mathrm{C} 15-\mathrm{C} 16-\mathrm{C} 17-\mathrm{C} 18$ & $-179.6(3)$ & $\mathrm{C} 15-\mathrm{C} 16-\mathrm{N} 4-\mathrm{Cu} 2$ & $-2.5(4)$ \\
\hline $\mathrm{C} 16-\mathrm{C} 17-\mathrm{C} 18-\mathrm{C} 19$ & $-0.9(5)$ & $\mathrm{N} 2-\mathrm{C} 10-\mathrm{O} 2-\mathrm{Cu} 2$ & $-9.7(5)$ \\
\hline $\mathrm{C} 17-\mathrm{C} 18-\mathrm{C} 19-\mathrm{C} 20$ & $0.7(6)$ & $\mathrm{C} 9-\mathrm{C} 10-\mathrm{O} 2-\mathrm{Cu} 2$ & $171.9(3)$ \\
\hline $\mathrm{C} 18-\mathrm{C} 19-\mathrm{C} 20-\mathrm{O} 4$ & $180.0(3)$ & $\mathrm{O} 8-\mathrm{N} 5-\mathrm{O} 6-\mathrm{Cu} 1$ & $-168.6(3)$ \\
\hline $\mathrm{C} 18-\mathrm{C} 19-\mathrm{C} 20-\mathrm{N} 4$ & $-0.1(6)$ & $\mathrm{O} 7-\mathrm{N} 5-\mathrm{O} 6-\mathrm{Cu} 1$ & $11.6(5)$ \\
\hline
\end{tabular}




\begin{tabular}{llll}
$\mathrm{O} 1-\mathrm{C} 1-\mathrm{N} 1-\mathrm{C} 5$ & $-179.2(3)$ & $\mathrm{O} 10-\mathrm{N} 6-\mathrm{O} 9-\mathrm{Cu} 1$ & $-18.1(5)$ \\
$\mathrm{C} 2-\mathrm{C} 1-\mathrm{N} 1-\mathrm{C} 5$ & $-0.4(5)$ & $\mathrm{O} 11-\mathrm{N} 6-\mathrm{O} 9-\mathrm{Cu} 1$ & $163.9(2)$ \\
$\mathrm{O} 1-\mathrm{C} 1-\mathrm{N} 1-\mathrm{Cu} 1$ & $-0.2(5)$ & $\mathrm{O} 9-\mathrm{N} 6-\mathrm{O} 10-\mathrm{Cu} 2$ & $20.0(5)$ \\
$\mathrm{C} 2-\mathrm{C} 1-\mathrm{N} 1-\mathrm{Cu} 1$ & $178.6(3)$ & $\mathrm{O} 11-\mathrm{N} 6-\mathrm{O} 10-\mathrm{Cu} 2$ & $-162.0(3)$ \\
\hline
\end{tabular}

Hydrogen-bond geometry $(\AA \stackrel{\circ}{\circ})$

\begin{tabular}{lllll}
\hline$D-\mathrm{H} \cdots A$ & $D-\mathrm{H}$ & $\mathrm{H} \cdots A$ & $D \cdots A$ & $D-\mathrm{H} \cdots A$ \\
\hline $\mathrm{O} 5-\mathrm{H} 15 \cdots \mathrm{O} 11^{\mathrm{i}}$ & $0.75(4)$ & $2.08(4)$ & $2.802(4)$ & $164(4)$ \\
$\mathrm{O} 1-\mathrm{H} 1 \cdots \mathrm{O} 6$ & $0.83(2)$ & $1.78(2)$ & $2.583(4)$ & $163(5)$ \\
$\mathrm{O} 3-\mathrm{H} 3 B \cdots \mathrm{O} 5$ & $0.83(2)$ & $1.70(2)$ & $2.510(4)$ & $164(5)$ \\
$\mathrm{O} 4-\mathrm{H} 4 B \cdots \mathrm{O} 2$ & $0.83(2)$ & $1.73(2)$ & $2.528(4)$ & $161(5)$ \\
\hline
\end{tabular}

Symmetry code: (i) $x+1, y, z$.

(II) Bis $\left(\mu_{3}\right.$-bipyridine-2,2'-diolato- $\left.\kappa^{3} O: N, N^{\prime}: O^{\prime}\right)$ tetrakis $\left(6,6^{\prime}\right.$-dihydroxybipyridine- $\left.\kappa^{2} N, N^{\prime}\right)$ tetrakis $(\mu$-hydroxido$\left.\kappa^{2} O: O^{\prime}\right)$ bis (methanol- $\left.\kappa O\right)$ tetrakis $\left(\mu\right.$-nitrato- $\left.\kappa^{2} O: O^{\prime}\right)$ hexacopper(II)

Crystal data

$\left[\mathrm{Cu}_{6}\left(\mathrm{C}_{10} \mathrm{H}_{6} \mathrm{~N}_{2} \mathrm{O}_{2}\right)_{2}\left(\mathrm{CH}_{4} \mathrm{O}\right)_{2}(\mathrm{OH})_{4}\left(\mathrm{NO}_{3}\right)_{4}\left(\mathrm{C}_{10} \mathrm{H}_{8} \mathrm{~N}_{2} \mathrm{O}_{2}\right)_{4}\right]$ $M_{r}=1886.53$

Triclinic, $P \overline{1}$

$a=10.2135(7) \AA$

$b=13.3707(8) \AA$

$c=14.0565(10) \AA$

$\alpha=64.591(4)^{\circ}$

$\beta=75.659(5)^{\circ}$

$\gamma=82.262(5)^{\circ}$

$V=1679.0(2) \AA^{3}$

\section{Data collection}

Bruker APEXII CCD

diffractometer

$\varphi$ and $\omega$ scans

Absorption correction: multi-scan

(TWINABS; Sheldrick, 2009)

$T_{\min }=0.622, T_{\max }=0.746$

20719 measured reflections

\section{Refinement}

Refinement on $F^{2}$

Least-squares matrix: full

$R\left[F^{2}>2 \sigma\left(F^{2}\right)\right]=0.044$

$w R\left(F^{2}\right)=0.119$

$S=1.00$

10920 reflections

529 parameters

2 restraints

Primary atom site location: structure-invariant direct methods
$Z=1$

$F(000)=954$

$D_{\mathrm{x}}=1.866 \mathrm{Mg} \mathrm{m}^{-3}$

Mo $K \alpha$ radiation, $\lambda=0.71073 \AA$

Cell parameters from 1117 reflections

$\theta=3.0-29.1^{\circ}$

$\mu=1.97 \mathrm{~mm}^{-1}$

$T=100 \mathrm{~K}$

Prism, green

$0.26 \times 0.22 \times 0.07 \mathrm{~mm}$

10920 independent reflections

7847 reflections with $I>2 \sigma(I)$

$R_{\text {int }}=0.068$

$\theta_{\max }=32.0^{\circ}, \theta_{\min }=1.6^{\circ}$

$h=-14 \rightarrow 14$

$k=-16 \rightarrow 19$

$l=0 \rightarrow 20$

Secondary atom site location: difference Fourier map

Hydrogen site location: mixed

$\mathrm{H}$ atoms treated by a mixture of independent and constrained refinement

$w=1 /\left[\sigma^{2}\left(F_{\mathrm{o}}^{2}\right)+(0.068 P)^{2}\right]$

where $P=\left(F_{\mathrm{o}}^{2}+2 F_{\mathrm{c}}{ }^{2}\right) / 3$

$(\Delta / \sigma)_{\max }=0.001$

$\Delta \rho_{\max }=0.90 \mathrm{e} \AA^{-3}$

$\Delta \rho_{\min }=-1.08$ e $\AA^{-3}$ 


\section{Special details}

Experimental. The crystal under investigation was found to be split with two major domains not related by any obvious twin operation. The orientation matrices for the two components were identified using the program Cell_Now, with the two components being related by a 2.9 degrees rotation about either the reciprocal axis $1.000-0.363-0.339$ or the real axis $1.000-0.178-0.265$ degree. The two components were integrated using Saint, resulting in in the following statistics:

17535 data (5769 unique) involve domain 1 only, mean I/sigma 8.617271 data (5689 unique) involve domain 2 only, mean I/sigma 8.234813 data (9811 unique) involve 2 domains, mean I/sigma 9.511 data (11 unique) involve 3 domains, mean I/sigma 8.74 data (2 unique) involve 4 domains, mean I/sigma 57.6

The exact correlation matrix was identified by the integration program was found to be Transforms h1.1(1)->h1.2(2) 1.003360 .02923 - 0.02720 - $0.018941 .02272-0.049030 .025200 .057470 .97055$.

The data were corrected for absorption using twinabs, and the structure was solved using direct methods with only the non-overlapping reflections of component 1 . The structure was refined using the hklf 5 routine with all reflections of component 1 (including the overlapping ones), resulting in a BASF value of 0.486 (1).

The $R_{\text {int }}$ value given is for all reflections and is based on agreement between observed single and composite intensities and those calculated from refined unique intensities and twin fractions (TWINABS (Sheldrick, 2009)).

Geometry. All e.s.d.'s (except the e.s.d. in the dihedral angle between two 1.s. planes) are estimated using the full covariance matrix. The cell e.s.d.'s are taken into account individually in the estimation of e.s.d.'s in distances, angles and torsion angles; correlations between e.s.d.'s in cell parameters are only used when they are defined by crystal symmetry. An approximate (isotropic) treatment of cell e.s.d.'s is used for estimating e.s.d.'s involving 1.s. planes.

Refinement. Refined as a 2-component twin.

Fractional atomic coordinates and isotropic or equivalent isotropic displacement parameters $\left(\AA^{2}\right)$

\begin{tabular}{|c|c|c|c|c|}
\hline & $x$ & $y$ & $z$ & $U_{\text {iso }} * / U_{\text {eq }}$ \\
\hline $\mathrm{Cu} 1$ & $0.41028(4)$ & $0.22335(3)$ & $0.75942(3)$ & $0.01004(9)$ \\
\hline $\mathrm{Cu} 2$ & $0.48719(4)$ & $0.28424(3)$ & $0.49234(3)$ & $0.01009(9)$ \\
\hline $\mathrm{Cu} 3$ & $0.79681(4)$ & $0.28550(3)$ & $0.32300(3)$ & 0.00978 (9) \\
\hline $\mathrm{O} 1$ & $0.6858(2)$ & $0.3553(2)$ & $0.66647(18)$ & $0.0149(5)$ \\
\hline H1 & 0.6187 & 0.3498 & 0.6447 & $0.022 *$ \\
\hline $\mathrm{O} 2$ & $0.1351(2)$ & $0.0820(2)$ & 0.89259 (19) & $0.0210(6)$ \\
\hline $\mathrm{H} 2 \mathrm{~A}$ & 0.1769 & 0.1133 & 0.8284 & $0.031 *$ \\
\hline $\mathrm{O} 3$ & $0.2766(2)$ & 0.17719 (19) & $0.70803(17)$ & $0.0108(4)$ \\
\hline $\mathrm{O} 4$ & $0.6689(2)$ & $0.3846(2)$ & $0.23790(17)$ & $0.0154(5)$ \\
\hline $\mathrm{O} 5$ & $0.8343(2)$ & $0.1430(2)$ & $0.56936(18)$ & $0.0162(5)$ \\
\hline H5 & 0.7765 & 0.1877 & 0.5382 & $0.024 *$ \\
\hline O6 & $0.8053(2)$ & $0.3901(2)$ & $0.06196(18)$ & $0.0174(5)$ \\
\hline H6 & 0.7504 & 0.3859 & 0.1189 & $0.026^{*}$ \\
\hline $\mathrm{O} 7$ & $0.2336(2)$ & $0.3684(2)$ & $0.77329(18)$ & $0.0169(5)$ \\
\hline O8 & $0.1064(2)$ & $0.5167(2)$ & 0.71865 (19) & $0.0172(5)$ \\
\hline O9 & $0.2815(2)$ & 0.49085 (19) & $0.60670(18)$ & $0.0166(5)$ \\
\hline $\mathrm{O} 10$ & $0.5101(3)$ & -0.0537 (2) & $0.6858(2)$ & $0.0244(6)$ \\
\hline O11 & $0.5594(2)$ & 0.0977 (2) & 0.68885 (19) & $0.0160(5)$ \\
\hline $\mathrm{O} 12$ & $0.4932(2)$ & $0.1038(2)$ & $0.55029(19)$ & $0.0164(5)$ \\
\hline $\mathrm{O} 13$ & $0.6824(2)$ & $0.28679(18)$ & $0.45612(17)$ & $0.0103(4)$ \\
\hline $\mathrm{H} 13 \mathrm{~A}$ & $0.695(4)$ & $0.347(2)$ & $0.455(3)$ & $0.015^{*}$ \\
\hline $\mathrm{O} 14$ & $0.4843(2)$ & 0.33310 (19) & $0.61349(17)$ & $0.0105(4)$ \\
\hline $\mathrm{H} 14 \mathrm{~A}$ & $0.432(3)$ & $0.387(2)$ & $0.598(3)$ & $0.016^{*}$ \\
\hline $\mathrm{O} 15$ & $0.7227(3)$ & $0.1269(2)$ & $0.3314(2)$ & $0.0262(6)$ \\
\hline H15 & $0.647(4)$ & $0.121(4)$ & $0.375(4)$ & $0.039 *$ \\
\hline
\end{tabular}




\begin{tabular}{|c|c|c|c|c|}
\hline N1 & $0.5541(2)$ & $0.2397(2)$ & $0.8244(2)$ & $0.0105(5)$ \\
\hline N2 & 0.3295 (3) & $0.1288(2)$ & 0.9159 (2) & $0.0111(5)$ \\
\hline N3 & $0.2886(2)$ & $0.2882(2)$ & $0.5293(2)$ & $0.0104(5)$ \\
\hline N4 & $0.4511(2)$ & $0.3683(2)$ & 0.3394 (2) & $0.0102(5)$ \\
\hline N5 & $0.9518(3)$ & 0.2047 (2) & $0.3927(2)$ & $0.0110(5)$ \\
\hline N6 & 0.9403 (3) & $0.3065(2)$ & 0.1862 (2) & $0.0108(5)$ \\
\hline N7 & 0.2064 (3) & $0.4580(2)$ & $0.7012(2)$ & $0.0125(5)$ \\
\hline N8 & $0.5208(3)$ & $0.0495(2)$ & $0.6423(2)$ & $0.0134(6)$ \\
\hline $\mathrm{C} 1$ & $0.6682(3)$ & 0.2959 (3) & $0.7701(3)$ & $0.0132(6)$ \\
\hline $\mathrm{C} 2$ & 0.7689 (3) & $0.2919(3)$ & 0.8239 (3) & $0.0148(7)$ \\
\hline $\mathrm{H} 2$ & 0.8502 & 0.3303 & 0.7848 & $0.018 *$ \\
\hline $\mathrm{C} 3$ & $0.7495(3)$ & $0.2321(3)$ & 0.9335 (3) & $0.0174(7)$ \\
\hline H3 & 0.8176 & 0.2287 & 0.9703 & $0.021 *$ \\
\hline $\mathrm{C} 4$ & 0.6293 (3) & $0.1761(3)$ & 0.9908 (3) & $0.0168(7)$ \\
\hline H4 & 0.6131 & 0.1359 & 1.0668 & $0.020 *$ \\
\hline $\mathrm{C} 5$ & 0.5354 (3) & $0.1810(3)$ & 0.9336 (3) & $0.0115(6)$ \\
\hline C6 & $0.4062(3)$ & $0.1227(3)$ & $0.9856(3)$ & $0.0124(7)$ \\
\hline $\mathrm{C} 7$ & $0.3656(3)$ & $0.0679(3)$ & 1.0958 (3) & $0.0157(7)$ \\
\hline H7 & 0.4210 & 0.0644 & 1.1423 & $0.019 *$ \\
\hline $\mathrm{C} 8$ & $0.2402(3)$ & $0.0176(3)$ & $1.1371(3)$ & $0.0177(7)$ \\
\hline H8 & 0.2100 & -0.0214 & 1.2127 & $0.021 *$ \\
\hline C9 & $0.1613(3)$ & $0.0243(3)$ & 1.0695 (3) & $0.0173(7)$ \\
\hline H9 & 0.0750 & -0.0079 & 1.0971 & $0.021 *$ \\
\hline $\mathrm{C} 10$ & $0.2101(3)$ & $0.0798(3)$ & 0.9580 (3) & $0.0148(7)$ \\
\hline C11 & $0.2146(3)$ & $0.2432(3)$ & $0.6310(3)$ & $0.0118(6)$ \\
\hline $\mathrm{C} 12$ & 0.0747 (3) & $0.2651(3)$ & $0.6532(3)$ & $0.0147(7)$ \\
\hline H12 & 0.0230 & 0.2328 & 0.7248 & $0.018 *$ \\
\hline $\mathrm{C} 13$ & 0.0135 (3) & $0.3340(3)$ & 0.5699 (3) & $0.0162(7)$ \\
\hline H13 & -0.0806 & 0.3515 & 0.5841 & $0.019 *$ \\
\hline C14 & 0.0893 (3) & $0.3782(3)$ & 0.4647 (3) & $0.0134(6)$ \\
\hline H14 & 0.0480 & 0.4249 & 0.4063 & $0.016^{*}$ \\
\hline C15 & $0.2260(3)$ & $0.3523(3)$ & 0.4478 (3) & $0.0107(6)$ \\
\hline $\mathrm{C} 16$ & $0.3161(3)$ & $0.3916(3)$ & 0.3392 (3) & $0.0107(6)$ \\
\hline $\mathrm{C} 17$ & $0.2675(3)$ & $0.4448(3)$ & $0.2472(3)$ & $0.0136(7)$ \\
\hline H17 & 0.1728 & 0.4556 & 0.2506 & $0.016^{*}$ \\
\hline $\mathrm{C} 18$ & 0.3584 (3) & $0.4833(3)$ & $0.1480(3)$ & $0.0170(7)$ \\
\hline H18 & 0.3268 & 0.5215 & 0.0829 & $0.020^{*}$ \\
\hline C19 & 0.4939 (3) & $0.4648(3)$ & 0.1461 (3) & $0.0155(7)$ \\
\hline H19 & 0.5575 & 0.4926 & 0.0795 & $0.019 *$ \\
\hline $\mathrm{C} 20$ & $0.5395(3)$ & $0.4043(3)$ & $0.2436(2)$ & $0.0124(6)$ \\
\hline $\mathrm{C} 21$ & $0.9476(3)$ & $0.1470(3)$ & 0.4981 (3) & $0.0132(6)$ \\
\hline $\mathrm{C} 22$ & $1.0618(3)$ & $0.0891(3)$ & $0.5372(3)$ & $0.0150(7)$ \\
\hline H22 & 1.0561 & 0.0473 & 0.6124 & $0.018^{*}$ \\
\hline $\mathrm{C} 23$ & $1.1811(3)$ & $0.0938(3)$ & $0.4656(3)$ & $0.0163(7)$ \\
\hline $\mathrm{H} 23$ & 1.2598 & 0.0562 & 0.4906 & $0.020^{*}$ \\
\hline $\mathrm{C} 24$ & $1.1870(3)$ & $0.1542(3)$ & $0.3551(3)$ & $0.0152(7)$ \\
\hline H24 & 1.2693 & 0.1577 & 0.3044 & $0.018^{*}$ \\
\hline $\mathrm{C} 25$ & $1.0716(3)$ & $0.2084(3)$ & $0.3209(3)$ & $0.0120(6)$ \\
\hline
\end{tabular}




\begin{tabular}{lllll}
$\mathrm{C} 26$ & $1.0664(3)$ & $0.2698(3)$ & $0.2063(3)$ & $0.0121(6)$ \\
$\mathrm{C} 27$ & $1.1783(3)$ & $0.2865(3)$ & $0.1232(3)$ & $0.0169(7)$ \\
$\mathrm{H} 27$ & 1.2658 & 0.2639 & 0.1385 & $0.020^{*}$ \\
$\mathrm{C} 28$ & $1.1625(3)$ & $0.3365(3)$ & $0.0176(3)$ & $0.0175(7)$ \\
$\mathrm{H} 28$ & 1.2391 & 0.3471 & -0.0398 & $0.021^{*}$ \\
$\mathrm{C} 29$ & $1.0370(3)$ & $0.3706(3)$ & $-0.0045(3)$ & $0.0165(7)$ \\
$\mathrm{H} 29$ & 1.0243 & 0.4034 & -0.0765 & $0.020^{*}$ \\
$\mathrm{C} 30$ & $0.9276(3)$ & $0.3557(3)$ & $0.0827(3)$ & $0.0136(7)$ \\
$\mathrm{C} 31$ & $0.7846(4)$ & $0.0935(4)$ & $0.2443(3)$ & $0.0308(9)$ \\
$\mathrm{H} 31 \mathrm{~A}$ & 0.8829 & 0.1003 & 0.2282 & $0.046^{*}$ \\
$\mathrm{H} 31 \mathrm{~B}$ & 0.7492 & 0.1413 & 0.1800 & $0.046^{*}$ \\
$\mathrm{H} 31 \mathrm{C}$ & 0.7636 & 0.0164 & 0.2659 & $0.046^{*}$ \\
\hline
\end{tabular}

Atomic displacement parameters $\left(\AA^{2}\right)$

\begin{tabular}{|c|c|c|c|c|c|c|}
\hline & $U^{11}$ & $U^{22}$ & $U^{33}$ & $U^{12}$ & $U^{13}$ & $U^{23}$ \\
\hline $\mathrm{Cu} 1$ & $0.00874(18)$ & $0.0129(2)$ & $0.00790(18)$ & $-0.00220(14)$ & $-0.00128(13)$ & $-0.00351(15)$ \\
\hline $\mathrm{Cu} 2$ & $0.00726(17)$ & $0.0145(2)$ & $0.00810(18)$ & $-0.00063(13)$ & $-0.00178(13)$ & $-0.00408(15)$ \\
\hline $\mathrm{Cu} 3$ & 0.00937 (18) & $0.0123(2)$ & $0.00782(18)$ & 0.00178 (14) & $-0.00247(13)$ & $-0.00451(15)$ \\
\hline $\mathrm{O} 1$ & $0.0088(10)$ & $0.0228(14)$ & $0.0133(11)$ & $-0.0060(9)$ & $-0.0021(8)$ & $-0.0062(10)$ \\
\hline $\mathrm{O} 2$ & $0.0149(12)$ & $0.0354(17)$ & $0.0115(12)$ & $-0.0138(11)$ & $-0.0006(9)$ & $-0.0061(12)$ \\
\hline $\mathrm{O} 3$ & $0.0098(10)$ & $0.0145(12)$ & $0.0072(10)$ & $-0.0033(8)$ & $-0.0019(8)$ & $-0.0028(9)$ \\
\hline $\mathrm{O} 4$ & $0.0101(11)$ & $0.0237(14)$ & $0.0083(10)$ & $0.0025(9)$ & $-0.0031(8)$ & $-0.0030(10)$ \\
\hline $\mathrm{O} 5$ & $0.0126(11)$ & $0.0195(13)$ & $0.0129(11)$ & $0.0018(9)$ & $-0.0040(9)$ & $-0.0033(10)$ \\
\hline O6 & $0.0135(11)$ & $0.0266(15)$ & $0.0115(11)$ & $0.0047(10)$ & $-0.0033(9)$ & $-0.0084(11)$ \\
\hline $\mathrm{O} 7$ & $0.0218(12)$ & $0.0130(13)$ & $0.0113(11)$ & $0.0030(9)$ & $-0.0016(9)$ & $-0.0027(10)$ \\
\hline $\mathrm{O} 8$ & $0.0127(11)$ & $0.0189(13)$ & $0.0217(13)$ & $0.0050(9)$ & $-0.0035(9)$ & $-0.0115(11)$ \\
\hline O9 & $0.0205(12)$ & $0.0140(12)$ & $0.0109(11)$ & $0.0007(9)$ & $0.0025(9)$ & $-0.0046(10)$ \\
\hline $\mathrm{O} 10$ & $0.0251(13)$ & $0.0103(13)$ & $0.0334(15)$ & $-0.0018(10)$ & $-0.0150(11)$ & $0.0000(12)$ \\
\hline $\mathrm{O} 11$ & $0.0130(11)$ & $0.0183(13)$ & $0.0194(12)$ & $-0.0009(9)$ & $-0.0042(9)$ & $-0.0098(11)$ \\
\hline $\mathrm{O} 12$ & $0.0171(12)$ & $0.0158(13)$ & $0.0159(12)$ & $0.0013(9)$ & $-0.0061(9)$ & $-0.0051(10)$ \\
\hline $\mathrm{O} 13$ & $0.0088(10)$ & $0.0124(12)$ & $0.0098(10)$ & $0.0007(8)$ & $-0.0029(8)$ & $-0.0047(9)$ \\
\hline $\mathrm{O} 14$ & $0.0096(10)$ & $0.0110(11)$ & $0.0111(10)$ & $0.0008(8)$ & $-0.0029(8)$ & $-0.0046(9)$ \\
\hline $\mathrm{O} 15$ & $0.0306(15)$ & $0.0195(15)$ & $0.0308(16)$ & $-0.0048(12)$ & $-0.0132(12)$ & $-0.0076(13)$ \\
\hline N1 & $0.0096(12)$ & $0.0133(14)$ & $0.0089(12)$ & $0.0012(10)$ & $-0.0016(10)$ & $-0.0055(11)$ \\
\hline N2 & $0.0104(12)$ & $0.0113(14)$ & $0.0097(12)$ & $-0.0029(10)$ & $-0.0018(10)$ & $-0.0022(11)$ \\
\hline N3 & $0.0096(12)$ & $0.0122(14)$ & $0.0117(13)$ & $-0.0002(9)$ & $-0.0020(10)$ & $-0.0074(11)$ \\
\hline N4 & $0.0100(12)$ & $0.0109(13)$ & $0.0103(12)$ & $0.0008(10)$ & $-0.0044(10)$ & $-0.0042(11)$ \\
\hline N5 & $0.0111(12)$ & $0.0104(13)$ & $0.0115(13)$ & $0.0007(10)$ & $-0.0023(10)$ & $-0.0049(11)$ \\
\hline N6 & $0.0130(13)$ & $0.0100(14)$ & $0.0099(12)$ & $0.0015(10)$ & $-0.0026(10)$ & $-0.0051(11)$ \\
\hline N7 & $0.0151(13)$ & $0.0130(14)$ & $0.0111(13)$ & $-0.0017(10)$ & $-0.0009(10)$ & $-0.0073(11)$ \\
\hline N8 & $0.0107(13)$ & $0.0095(14)$ & $0.0179(14)$ & $-0.0001(10)$ & $-0.0046(11)$ & $-0.0029(12)$ \\
\hline $\mathrm{C} 1$ & $0.0140(15)$ & $0.0133(17)$ & $0.0138(15)$ & $0.0006(12)$ & $-0.0024(12)$ & $-0.0077(13)$ \\
\hline $\mathrm{C} 2$ & $0.0085(14)$ & $0.0195(19)$ & $0.0196(17)$ & $-0.0022(12)$ & $-0.0028(12)$ & $-0.0108(15)$ \\
\hline $\mathrm{C} 3$ & $0.0151(16)$ & $0.0193(19)$ & $0.0204(18)$ & $-0.0002(13)$ & $-0.0081(13)$ & $-0.0085(15)$ \\
\hline $\mathrm{C} 4$ & $0.0187(17)$ & $0.021(2)$ & $0.0120(15)$ & $0.0006(14)$ & $-0.0055(13)$ & $-0.0075(14)$ \\
\hline $\mathrm{C} 5$ & $0.0102(14)$ & $0.0146(17)$ & $0.0126(15)$ & $0.0018(12)$ & $-0.0039(12)$ & $-0.0082(13)$ \\
\hline C6 & $0.0125(15)$ & $0.0115(17)$ & $0.0118(15)$ & $0.0019(12)$ & $-0.0033(12)$ & $-0.0039(13)$ \\
\hline
\end{tabular}




$\begin{array}{lllllll}\text { C7 } & 0.0185(17) & 0.0177(19) & 0.0101(15) & 0.0015(13) & -0.0032(12) & -0.0055(14) \\ \text { C8 } & 0.0225(18) & 0.0147(18) & 0.0115(15) & -0.0025(13) & 0.0008(13) & -0.0031(14) \\ \text { C9 } & 0.0147(16) & 0.020(2) & 0.0141(16) & -0.0062(13) & 0.0032(13) & -0.0059(15) \\ \text { C10 } & 0.0126(15) & 0.0181(18) & 0.0141(16) & -0.0011(13) & -0.0026(12) & -0.0069(14) \\ \text { C11 } & 0.0106(14) & 0.0118(16) & 0.0122(15) & -0.0034(11) & -0.0001(11) & -0.0045(13) \\ \text { C12 } & 0.0116(15) & 0.0191(18) & 0.0152(16) & -0.0013(12) & -0.0005(12) & -0.0097(14) \\ \text { C13 } & 0.0102(14) & 0.0206(18) & 0.0197(17) & 0.0006(12) & -0.0013(12) & -0.0113(15) \\ \text { C14 } & 0.0130(15) & 0.0147(17) & 0.0136(15) & 0.0017(12) & -0.0051(12) & -0.0061(13) \\ \text { C15 } & 0.0101(14) & 0.0117(16) & 0.0123(15) & -0.0001(11) & -0.0043(11) & -0.0059(13) \\ \text { C16 } & 0.0118(14) & 0.0093(15) & 0.0136(15) & 0.0012(11) & -0.0039(11) & -0.0069(13) \\ \text { C17 } & 0.0139(15) & 0.0135(17) & 0.0148(16) & 0.0030(12) & -0.0055(12) & -0.0067(14) \\ \text { C18 } & 0.0197(17) & 0.021(2) & 0.0119(15) & 0.0066(14) & -0.0076(13) & -0.0075(14) \\ \text { C19 } & 0.0161(16) & 0.0209(19) & 0.0069(14) & 0.0047(13) & -0.0035(12) & -0.0042(14) \\ \text { C20 } & 0.0129(15) & 0.0151(17) & 0.0091(14) & 0.0026(12) & -0.0034(11) & -0.0051(13) \\ \text { C21 } & 0.0148(15) & 0.0116(16) & 0.0143(15) & -0.0009(12) & -0.0033(12) & -0.0059(13) \\ \text { C22 } & 0.0175(16) & 0.0135(17) & 0.0139(15) & 0.0004(12) & -0.0079(12) & -0.0035(13) \\ \text { C23 } & 0.0151(16) & 0.0149(17) & 0.0189(17) & 0.0051(12) & -0.0077(13) & -0.0063(14) \\ \text { C24 } & 0.0112(15) & 0.0180(18) & 0.0194(17) & 0.0028(12) & -0.0036(12) & -0.0112(15) \\ \text { C25 } & 0.0128(15) & 0.0114(16) & 0.0138(15) & 0.0025(11) & -0.0025(12) & -0.0083(13) \\ \text { C26 } & 0.0125(15) & 0.0116(16) & 0.0167(16) & 0.0014(12) & -0.0053(12) & -0.0093(13) \\ \text { C27 } & 0.0137(16) & 0.0205(19) & 0.0196(17) & 0.0022(13) & -0.0031(13) & -0.0121(15) \\ \text { C28 } & 0.0150(16) & 0.021(2) & 0.0174(16) & -0.0005(13) & 0.0017(13) & -0.0118(15) \\ \text { C29 } & 0.0191(17) & 0.0209(19) & 0.0107(15) & 0.0016(14) & -0.0031(12) & -0.0083(14) \\ \text { C30 } & 0.0149(16) & 0.0116(17) & 0.0136(15) & 0.0032(12) & -0.0021(12) & -0.0060(13) \\ \text { C31 } & 0.036(2) & 0.030(2) & 0.032(2) & -0.0003(18) & -0.0100(18) & -0.016(2) \\ & & & & & & \end{array}$

Geometric parameters $\left(A,{ }^{\circ}\right)$

\begin{tabular}{llll}
\hline $\mathrm{Cu} 1-\mathrm{O} 3$ & $1.966(2)$ & $\mathrm{C} 1-\mathrm{C} 2$ & $1.403(5)$ \\
$\mathrm{Cu} 1-\mathrm{O} 14$ & $1.970(2)$ & $\mathrm{C} 2-\mathrm{C} 3$ & $1.373(5)$ \\
$\mathrm{Cu} 1-\mathrm{N} 1$ & $1.991(3)$ & $\mathrm{C} 2-\mathrm{H} 2$ & 0.9500 \\
$\mathrm{Cu} 1-\mathrm{N} 2$ & $2.028(3)$ & $\mathrm{C} 3-\mathrm{C} 4$ & $1.399(5)$ \\
$\mathrm{Cu} 1-\mathrm{O} 11$ & $2.474(2)$ & $\mathrm{C} 3-\mathrm{H} 3$ & 0.9500 \\
$\mathrm{Cu} 1-\mathrm{O} 7$ & $\mathrm{C} 4-\mathrm{C} 5$ & $1.374(5)$ \\
$\mathrm{Cu} 2-\mathrm{O} 13$ & $2.503(2)$ & $\mathrm{C} 4-\mathrm{H} 4$ & 0.9500 \\
$\mathrm{Cu} 2-\mathrm{N} 3$ & $1.933(2)$ & $\mathrm{C} 5-\mathrm{C} 6$ & $1.481(5)$ \\
$\mathrm{Cu} 2-\mathrm{N} 4$ & $1.964(2)$ & $\mathrm{C} 6-\mathrm{C} 7$ & $1.377(4)$ \\
$\mathrm{Cu} 2-\mathrm{O} 14$ & $2.056(3)$ & $\mathrm{C} 7-\mathrm{C} 8$ & $1.399(5)$ \\
$\mathrm{Cu} 2-\mathrm{O} 12$ & $2.062(2)$ & $\mathrm{C} 7-\mathrm{H} 7$ & 0.9500 \\
$\mathrm{Cu} 3-\mathrm{O} 13$ & $2.188(2)$ & $\mathrm{C} 8-\mathrm{C} 9$ & $1.358(5)$ \\
$\mathrm{Cu} 3-\mathrm{O} 4$ & $1.951(2)$ & $\mathrm{C} 8-\mathrm{H} 8$ & 0.9500 \\
$\mathrm{Cu} 3-\mathrm{N} 5$ & $1.960(2)$ & $\mathrm{C} 9-\mathrm{C} 10$ & $1.404(5)$ \\
$\mathrm{Cu} 3-\mathrm{N} 6$ & $2.008(3)$ & $\mathrm{C} 11-\mathrm{H} 9$ & 0.9500 \\
$\mathrm{Cu} 3-\mathrm{C} 12$ & $1.404(4)$ \\
$\mathrm{O} 1-\mathrm{C} 1$ & $2.044(2)$ & $\mathrm{C} 12-\mathrm{C} 13$ & $1.376(5)$ \\
$\mathrm{O} 1-\mathrm{H} 1$ & $2.292(3)$ & $\mathrm{C} 12-\mathrm{H} 12$ & 0.9500 \\
$\mathrm{O} 2-\mathrm{C} 10$ & $1.304(4)$ & $\mathrm{C} 13-\mathrm{C} 14$ & $1.393(4)$ \\
$\mathrm{O} 2-\mathrm{H} 2 \mathrm{~A}$ & 0.8400 & $\mathrm{C} 13-\mathrm{H} 13$ & 0.9500 \\
& $1.324(4)$ & &
\end{tabular}




\begin{tabular}{|c|c|c|c|}
\hline $\mathrm{O} 3-\mathrm{C} 11$ & $1.312(4)$ & $\mathrm{C} 14-\mathrm{C} 15$ & $1.380(4)$ \\
\hline $\mathrm{O} 4-\mathrm{C} 20$ & $1.302(4)$ & $\mathrm{C} 14-\mathrm{H} 14$ & 0.9500 \\
\hline $\mathrm{O} 5-\mathrm{C} 21$ & $1.321(4)$ & $\mathrm{C} 15-\mathrm{C} 16$ & $1.483(4)$ \\
\hline $\mathrm{O} 5-\mathrm{H} 5$ & 0.8400 & $\mathrm{C} 16-\mathrm{C} 17$ & $1.363(4)$ \\
\hline $\mathrm{O} 6-\mathrm{C} 30$ & $1.330(4)$ & $\mathrm{C} 17-\mathrm{C} 18$ & $1.394(5)$ \\
\hline O6-H6 & 0.8400 & $\mathrm{C} 17-\mathrm{H} 17$ & 0.9500 \\
\hline $\mathrm{O} 7-\mathrm{N} 7$ & $1.244(3)$ & $\mathrm{C} 18-\mathrm{C} 19$ & $1.369(4)$ \\
\hline O8-N7 & $1.243(3)$ & $\mathrm{C} 18-\mathrm{H} 18$ & 0.9500 \\
\hline $\mathrm{O} 9-\mathrm{N} 7$ & $1.277(3)$ & $\mathrm{C} 19-\mathrm{C} 20$ & $1.418(4)$ \\
\hline $\mathrm{O} 10-\mathrm{N} 8$ & $1.255(4)$ & C19-H19 & 0.9500 \\
\hline $\mathrm{O} 11-\mathrm{N} 8$ & $1.247(4)$ & $\mathrm{C} 21-\mathrm{C} 22$ & $1.404(4)$ \\
\hline $\mathrm{O} 12-\mathrm{N} 8$ & $1.265(4)$ & $\mathrm{C} 22-\mathrm{C} 23$ & $1.364(4)$ \\
\hline $\mathrm{O} 13-\mathrm{H} 13 \mathrm{~A}$ & 0.828 (19) & $\mathrm{C} 22-\mathrm{H} 22$ & 0.9500 \\
\hline $\mathrm{O} 14-\mathrm{H} 14 \mathrm{~A}$ & $0.823(18)$ & $\mathrm{C} 23-\mathrm{C} 24$ & $1.400(5)$ \\
\hline $\mathrm{O} 15-\mathrm{C} 31$ & $1.452(5)$ & $\mathrm{C} 23-\mathrm{H} 23$ & 0.9500 \\
\hline $\mathrm{O} 15-\mathrm{H} 15$ & $0.85(4)$ & $\mathrm{C} 24-\mathrm{C} 25$ & $1.377(4)$ \\
\hline $\mathrm{N} 1-\mathrm{C} 1$ & $1.345(4)$ & $\mathrm{C} 24-\mathrm{H} 24$ & 0.9500 \\
\hline $\mathrm{N} 1-\mathrm{C} 5$ & $1.368(4)$ & $\mathrm{C} 25-\mathrm{C} 26$ & $1.472(4)$ \\
\hline $\mathrm{N} 2-\mathrm{C} 10$ & $1.338(4)$ & $\mathrm{C} 26-\mathrm{C} 27$ & $1.379(4)$ \\
\hline $\mathrm{N} 2-\mathrm{C} 6$ & $1.369(4)$ & $\mathrm{C} 27-\mathrm{C} 28$ & $1.385(5)$ \\
\hline $\mathrm{N} 3-\mathrm{C} 11$ & $1.352(4)$ & $\mathrm{C} 27-\mathrm{H} 27$ & 0.9500 \\
\hline N3 $-\mathrm{C} 15$ & $1.352(4)$ & $\mathrm{C} 28-\mathrm{C} 29$ & $1.365(5)$ \\
\hline $\mathrm{N} 4-\mathrm{C} 20$ & $1.349(4)$ & $\mathrm{C} 28-\mathrm{H} 28$ & 0.9500 \\
\hline $\mathrm{N} 4-\mathrm{C} 16$ & $1.373(4)$ & $\mathrm{C} 29-\mathrm{C} 30$ & $1.401(4)$ \\
\hline N5-C21 & $1.337(4)$ & $\mathrm{C} 29-\mathrm{H} 29$ & 0.9500 \\
\hline N5-C25 & $1.372(4)$ & $\mathrm{C} 31-\mathrm{H} 31 \mathrm{~A}$ & 0.9800 \\
\hline N6-C30 & $1.346(4)$ & $\mathrm{C} 31-\mathrm{H} 31 \mathrm{~B}$ & 0.9800 \\
\hline N6-C26 & $1.368(4)$ & $\mathrm{C} 31-\mathrm{H} 31 \mathrm{C}$ & 0.9800 \\
\hline $\mathrm{O} 3-\mathrm{Cu} 1-\mathrm{O} 14$ & $92.09(9)$ & $\mathrm{C} 5-\mathrm{C} 4-\mathrm{H} 4$ & 121.0 \\
\hline $\mathrm{O} 3-\mathrm{Cu} 1-\mathrm{N} 1$ & $169.20(10)$ & $\mathrm{C} 3-\mathrm{C} 4-\mathrm{H} 4$ & 121.0 \\
\hline $\mathrm{O} 14-\mathrm{Cu} 1-\mathrm{N} 1$ & $94.74(10)$ & $\mathrm{N} 1-\mathrm{C} 5-\mathrm{C} 4$ & $122.7(3)$ \\
\hline $\mathrm{O} 3-\mathrm{Cu} 1-\mathrm{N} 2$ & $92.42(10)$ & $\mathrm{N} 1-\mathrm{C} 5-\mathrm{C} 6$ & $114.8(3)$ \\
\hline $\mathrm{O} 14-\mathrm{Cu} 1-\mathrm{N} 2$ & $171.80(10)$ & $\mathrm{C} 4-\mathrm{C} 5-\mathrm{C} 6$ & $122.6(3)$ \\
\hline $\mathrm{N} 1-\mathrm{Cu} 1-\mathrm{N} 2$ & $81.86(11)$ & $\mathrm{N} 2-\mathrm{C} 6-\mathrm{C} 7$ & $122.7(3)$ \\
\hline $\mathrm{O} 3-\mathrm{Cu} 1-\mathrm{O} 11$ & $81.68(8)$ & $\mathrm{N} 2-\mathrm{C} 6-\mathrm{C} 5$ & $114.9(3)$ \\
\hline $\mathrm{O} 14-\mathrm{Cu} 1-\mathrm{O} 11$ & $81.22(8)$ & $\mathrm{C} 7-\mathrm{C} 6-\mathrm{C} 5$ & $122.4(3)$ \\
\hline $\mathrm{N} 1-\mathrm{Cu} 1-\mathrm{O} 11$ & $91.10(9)$ & $\mathrm{C} 6-\mathrm{C} 7-\mathrm{C} 8$ & $118.1(3)$ \\
\hline $\mathrm{N} 2-\mathrm{Cu} 1-\mathrm{O} 11$ & $106.22(9)$ & $\mathrm{C} 6-\mathrm{C} 7-\mathrm{H} 7$ & 121.0 \\
\hline $\mathrm{O} 3-\mathrm{Cu} 1-\mathrm{O} 7$ & $83.89(9)$ & $\mathrm{C} 8-\mathrm{C} 7-\mathrm{H} 7$ & 121.0 \\
\hline $\mathrm{O} 14-\mathrm{Cu} 1-\mathrm{O} 7$ & $86.60(8)$ & $\mathrm{C} 9-\mathrm{C} 8-\mathrm{C} 7$ & $120.3(3)$ \\
\hline $\mathrm{N} 1-\mathrm{Cu} 1-\mathrm{O} 7$ & $104.84(9)$ & $\mathrm{C} 9-\mathrm{C} 8-\mathrm{H} 8$ & 119.8 \\
\hline $\mathrm{N} 2-\mathrm{Cu} 1-\mathrm{O} 7$ & $87.07(9)$ & $\mathrm{C} 7-\mathrm{C} 8-\mathrm{H} 8$ & 119.8 \\
\hline $\mathrm{O} 11-\mathrm{Cu} 1-\mathrm{O} 7$ & $160.71(8)$ & $\mathrm{C} 8-\mathrm{C} 9-\mathrm{C} 10$ & $118.6(3)$ \\
\hline $\mathrm{O} 13-\mathrm{Cu} 2-\mathrm{N} 3$ & $177.44(10)$ & $\mathrm{C} 8-\mathrm{C} 9-\mathrm{H} 9$ & 120.7 \\
\hline $\mathrm{O} 13-\mathrm{Cu} 2-\mathrm{N} 4$ & $98.59(10)$ & $\mathrm{C} 10-\mathrm{C} 9-\mathrm{H} 9$ & 120.7 \\
\hline $\mathrm{N} 3-\mathrm{Cu} 2-\mathrm{N} 4$ & $81.24(10)$ & $\mathrm{O} 2-\mathrm{C} 10-\mathrm{N} 2$ & $119.2(3)$ \\
\hline $\mathrm{O} 13-\mathrm{Cu} 2-\mathrm{O} 14$ & $90.04(9)$ & $\mathrm{O} 2-\mathrm{C} 10-\mathrm{C} 9$ & $118.2(3)$ \\
\hline
\end{tabular}




\begin{tabular}{|c|c|c|c|}
\hline $\mathrm{N} 3-\mathrm{Cu} 2-\mathrm{O} 14$ & $88.22(9)$ & $\mathrm{N} 2-\mathrm{C} 10-\mathrm{C} 9$ & $122.6(3)$ \\
\hline $\mathrm{N} 4-\mathrm{Cu} 2-\mathrm{O} 14$ & $132.65(10)$ & $\mathrm{O} 3-\mathrm{C} 11-\mathrm{N} 3$ & $118.3(3)$ \\
\hline $\mathrm{O} 13-\mathrm{Cu} 2-\mathrm{O} 12$ & $91.19(9)$ & $\mathrm{O} 3-\mathrm{C} 11-\mathrm{C} 12$ & $121.0(3)$ \\
\hline $\mathrm{N} 3-\mathrm{Cu} 2-\mathrm{O} 12$ & $91.20(10)$ & $\mathrm{N} 3-\mathrm{C} 11-\mathrm{C} 12$ & $120.8(3)$ \\
\hline $\mathrm{N} 4-\mathrm{Cu} 2-\mathrm{O} 12$ & $114.24(10)$ & $\mathrm{C} 13-\mathrm{C} 12-\mathrm{C} 11$ & $119.1(3)$ \\
\hline $\mathrm{O} 14-\mathrm{Cu} 2-\mathrm{O} 12$ & $112.01(9)$ & $\mathrm{C} 13-\mathrm{C} 12-\mathrm{H} 12$ & 120.5 \\
\hline $\mathrm{O} 13-\mathrm{Cu} 3-\mathrm{O} 4$ & $91.56(9)$ & $\mathrm{C} 11-\mathrm{C} 12-\mathrm{H} 12$ & 120.5 \\
\hline $\mathrm{O} 13-\mathrm{Cu} 3-\mathrm{N} 5$ & $93.98(10)$ & $\mathrm{C} 12-\mathrm{C} 13-\mathrm{C} 14$ & $120.1(3)$ \\
\hline $\mathrm{O} 4-\mathrm{Cu} 3-\mathrm{N} 5$ & $169.81(11)$ & $\mathrm{C} 12-\mathrm{C} 13-\mathrm{H} 13$ & 119.9 \\
\hline $\mathrm{O} 13-\mathrm{Cu} 3-\mathrm{N} 6$ & $168.03(10)$ & $\mathrm{C} 14-\mathrm{C} 13-\mathrm{H} 13$ & 119.9 \\
\hline $\mathrm{O} 4-\mathrm{Cu} 3-\mathrm{N} 6$ & $91.13(10)$ & $\mathrm{C} 15-\mathrm{C} 14-\mathrm{C} 13$ & $118.1(3)$ \\
\hline $\mathrm{N} 5-\mathrm{Cu} 3-\mathrm{N} 6$ & $81.74(10)$ & $\mathrm{C} 15-\mathrm{C} 14-\mathrm{H} 14$ & 120.9 \\
\hline $\mathrm{O} 13-\mathrm{Cu} 3-\mathrm{O} 15$ & $98.82(10)$ & $\mathrm{C} 13-\mathrm{C} 14-\mathrm{H} 14$ & 120.9 \\
\hline $\mathrm{O} 4-\mathrm{Cu} 3-\mathrm{O} 15$ & $94.90(10)$ & $\mathrm{N} 3-\mathrm{C} 15-\mathrm{C} 14$ & $122.5(3)$ \\
\hline $\mathrm{N} 5-\mathrm{Cu} 3-\mathrm{O} 15$ & $92.70(10)$ & $\mathrm{N} 3-\mathrm{C} 15-\mathrm{C} 16$ & $114.5(3)$ \\
\hline $\mathrm{N} 6-\mathrm{Cu} 3-\mathrm{O} 15$ & $92.58(11)$ & $\mathrm{C} 14-\mathrm{C} 15-\mathrm{C} 16$ & $122.9(3)$ \\
\hline $\mathrm{C} 1-\mathrm{O} 1-\mathrm{H} 1$ & 109.5 & $\mathrm{C} 17-\mathrm{C} 16-\mathrm{N} 4$ & $123.2(3)$ \\
\hline $\mathrm{C} 10-\mathrm{O} 2-\mathrm{H} 2 \mathrm{~A}$ & 109.5 & $\mathrm{C} 17-\mathrm{C} 16-\mathrm{C} 15$ & $122.3(3)$ \\
\hline $\mathrm{C} 11-\mathrm{O} 3-\mathrm{Cu} 1$ & $125.2(2)$ & $\mathrm{N} 4-\mathrm{C} 16-\mathrm{C} 15$ & $114.5(3)$ \\
\hline $\mathrm{C} 20-\mathrm{O} 4-\mathrm{Cu} 3$ & $140.7(2)$ & $\mathrm{C} 16-\mathrm{C} 17-\mathrm{C} 18$ & $119.2(3)$ \\
\hline $\mathrm{C} 21-\mathrm{O} 5-\mathrm{H} 5$ & 109.5 & $\mathrm{C} 16-\mathrm{C} 17-\mathrm{H} 17$ & 120.4 \\
\hline $\mathrm{C} 30-\mathrm{O} 6-\mathrm{H} 6$ & 109.5 & $\mathrm{C} 18-\mathrm{C} 17-\mathrm{H} 17$ & 120.4 \\
\hline $\mathrm{N} 7-\mathrm{O} 7-\mathrm{Cu} 1$ & $128.98(17)$ & $\mathrm{C} 19-\mathrm{C} 18-\mathrm{C} 17$ & $118.7(3)$ \\
\hline $\mathrm{N} 8-\mathrm{O} 11-\mathrm{Cu} 1$ & $123.07(18)$ & $\mathrm{C} 19-\mathrm{C} 18-\mathrm{H} 18$ & 120.6 \\
\hline $\mathrm{N} 8-\mathrm{O} 12-\mathrm{Cu} 2$ & $115.9(2)$ & $\mathrm{C} 17-\mathrm{C} 18-\mathrm{H} 18$ & 120.6 \\
\hline $\mathrm{Cu} 2-\mathrm{O} 13-\mathrm{Cu} 3$ & $125.46(12)$ & $\mathrm{C} 18-\mathrm{C} 19-\mathrm{C} 20$ & $120.1(3)$ \\
\hline $\mathrm{Cu} 2-\mathrm{O} 13-\mathrm{H} 13 \mathrm{~A}$ & $101(3)$ & $\mathrm{C} 18-\mathrm{C} 19-\mathrm{H} 19$ & 120.0 \\
\hline $\mathrm{Cu} 3-\mathrm{O} 13-\mathrm{H} 13 \mathrm{~A}$ & $105(3)$ & $\mathrm{C} 20-\mathrm{C} 19-\mathrm{H} 19$ & 120.0 \\
\hline $\mathrm{Cu} 1-\mathrm{O} 14-\mathrm{Cu} 2$ & $114.21(11)$ & $\mathrm{O} 4-\mathrm{C} 20-\mathrm{N} 4$ & $121.0(3)$ \\
\hline $\mathrm{Cu} 1-\mathrm{O} 14-\mathrm{H} 14 \mathrm{~A}$ & $109(3)$ & $\mathrm{O} 4-\mathrm{C} 20-\mathrm{C} 19$ & $118.2(3)$ \\
\hline $\mathrm{Cu} 2-\mathrm{O} 14-\mathrm{H} 14 \mathrm{~A}$ & $101(3)$ & $\mathrm{N} 4-\mathrm{C} 20-\mathrm{C} 19$ & $120.9(3)$ \\
\hline $\mathrm{C} 31-\mathrm{O} 15-\mathrm{Cu} 3$ & $117.3(2)$ & $\mathrm{O} 5-\mathrm{C} 21-\mathrm{N} 5$ & $120.4(3)$ \\
\hline $\mathrm{C} 31-\mathrm{O} 15-\mathrm{H} 15$ & $137(3)$ & $\mathrm{O} 5-\mathrm{C} 21-\mathrm{C} 22$ & $117.6(3)$ \\
\hline $\mathrm{Cu} 3-\mathrm{O} 15-\mathrm{H} 15$ & $100(3)$ & $\mathrm{N} 5-\mathrm{C} 21-\mathrm{C} 22$ & $122.0(3)$ \\
\hline $\mathrm{C} 1-\mathrm{N} 1-\mathrm{C} 5$ & $119.1(3)$ & $\mathrm{C} 23-\mathrm{C} 22-\mathrm{C} 21$ & $118.9(3)$ \\
\hline $\mathrm{C} 1-\mathrm{N} 1-\mathrm{Cu} 1$ & $125.9(2)$ & $\mathrm{C} 23-\mathrm{C} 22-\mathrm{H} 22$ & 120.6 \\
\hline $\mathrm{C} 5-\mathrm{N} 1-\mathrm{Cu} 1$ & $114.8(2)$ & $\mathrm{C} 21-\mathrm{C} 22-\mathrm{H} 22$ & 120.6 \\
\hline $\mathrm{C} 10-\mathrm{N} 2-\mathrm{C} 6$ & $117.8(3)$ & $\mathrm{C} 22-\mathrm{C} 23-\mathrm{C} 24$ & $119.7(3)$ \\
\hline $\mathrm{C} 10-\mathrm{N} 2-\mathrm{Cu} 1$ & $128.6(2)$ & $\mathrm{C} 22-\mathrm{C} 23-\mathrm{H} 23$ & 120.1 \\
\hline $\mathrm{C} 6-\mathrm{N} 2-\mathrm{Cu} 1$ & $113.5(2)$ & $\mathrm{C} 24-\mathrm{C} 23-\mathrm{H} 23$ & 120.1 \\
\hline $\mathrm{C} 11-\mathrm{N} 3-\mathrm{C} 15$ & $119.4(3)$ & $\mathrm{C} 25-\mathrm{C} 24-\mathrm{C} 23$ & $119.0(3)$ \\
\hline $\mathrm{C} 11-\mathrm{N} 3-\mathrm{Cu} 2$ & $124.0(2)$ & $\mathrm{C} 25-\mathrm{C} 24-\mathrm{H} 24$ & 120.5 \\
\hline $\mathrm{C} 15-\mathrm{N} 3-\mathrm{Cu} 2$ & $116.1(2)$ & $\mathrm{C} 23-\mathrm{C} 24-\mathrm{H} 24$ & 120.5 \\
\hline $\mathrm{C} 20-\mathrm{N} 4-\mathrm{C} 16$ & $117.8(3)$ & $\mathrm{N} 5-\mathrm{C} 25-\mathrm{C} 24$ & $121.4(3)$ \\
\hline $\mathrm{C} 20-\mathrm{N} 4-\mathrm{Cu} 2$ & $129.6(2)$ & $\mathrm{N} 5-\mathrm{C} 25-\mathrm{C} 26$ & $115.6(3)$ \\
\hline $\mathrm{C} 16-\mathrm{N} 4-\mathrm{Cu} 2$ & $112.6(2)$ & $\mathrm{C} 24-\mathrm{C} 25-\mathrm{C} 26$ & $123.0(3)$ \\
\hline $\mathrm{C} 21-\mathrm{N} 5-\mathrm{C} 25$ & $118.9(3)$ & $\mathrm{N} 6-\mathrm{C} 26-\mathrm{C} 27$ & $121.2(3)$ \\
\hline $\mathrm{C} 21-\mathrm{N} 5-\mathrm{Cu} 3$ & $127.1(2)$ & $\mathrm{N} 6-\mathrm{C} 26-\mathrm{C} 25$ & $115.3(3)$ \\
\hline
\end{tabular}




\begin{tabular}{|c|c|c|c|}
\hline $\mathrm{C} 25-\mathrm{N} 5-\mathrm{Cu} 3$ & $113.9(2)$ & $\mathrm{C} 27-\mathrm{C} 26-\mathrm{C} 25$ & $123.5(3)$ \\
\hline $\mathrm{C} 30-\mathrm{N} 6-\mathrm{C} 26$ & $118.0(3)$ & $\mathrm{C} 26-\mathrm{C} 27-\mathrm{C} 28$ & $119.7(3)$ \\
\hline $\mathrm{C} 30-\mathrm{N} 6-\mathrm{Cu} 3$ & $129.0(2)$ & $\mathrm{C} 26-\mathrm{C} 27-\mathrm{H} 27$ & 120.2 \\
\hline $\mathrm{C} 26-\mathrm{N} 6-\mathrm{Cu} 3$ & $112.9(2)$ & $\mathrm{C} 28-\mathrm{C} 27-\mathrm{H} 27$ & 120.2 \\
\hline $\mathrm{O} 8-\mathrm{N} 7-\mathrm{O} 7$ & $121.4(3)$ & $\mathrm{C} 29-\mathrm{C} 28-\mathrm{C} 27$ & $120.2(3)$ \\
\hline $\mathrm{O} 8-\mathrm{N} 7-\mathrm{O} 9$ & $118.7(3)$ & $\mathrm{C} 29-\mathrm{C} 28-\mathrm{H} 28$ & 119.9 \\
\hline $\mathrm{O} 7-\mathrm{N} 7-\mathrm{O} 9$ & $119.9(2)$ & $\mathrm{C} 27-\mathrm{C} 28-\mathrm{H} 28$ & 119.9 \\
\hline $\mathrm{O} 11-\mathrm{N} 8-\mathrm{O} 10$ & $120.4(3)$ & $\mathrm{C} 28-\mathrm{C} 29-\mathrm{C} 30$ & $117.8(3)$ \\
\hline $\mathrm{O} 11-\mathrm{N} 8-\mathrm{O} 12$ & $120.5(3)$ & $\mathrm{C} 28-\mathrm{C} 29-\mathrm{H} 29$ & 121.1 \\
\hline $\mathrm{O} 10-\mathrm{N} 8-\mathrm{O} 12$ & $119.1(3)$ & $\mathrm{C} 30-\mathrm{C} 29-\mathrm{H} 29$ & 121.1 \\
\hline $\mathrm{O} 1-\mathrm{C} 1-\mathrm{N} 1$ & $120.0(3)$ & $\mathrm{O} 6-\mathrm{C} 30-\mathrm{N} 6$ & $118.7(3)$ \\
\hline $\mathrm{O} 1-\mathrm{C} 1-\mathrm{C} 2$ & $119.4(3)$ & $\mathrm{O} 6-\mathrm{C} 30-\mathrm{C} 29$ & $118.2(3)$ \\
\hline $\mathrm{N} 1-\mathrm{C} 1-\mathrm{C} 2$ & $120.6(3)$ & $\mathrm{N} 6-\mathrm{C} 30-\mathrm{C} 29$ & $123.1(3)$ \\
\hline $\mathrm{C} 3-\mathrm{C} 2-\mathrm{C} 1$ & $119.8(3)$ & $\mathrm{O} 15-\mathrm{C} 31-\mathrm{H} 31 \mathrm{~A}$ & 109.5 \\
\hline $\mathrm{C} 3-\mathrm{C} 2-\mathrm{H} 2$ & 120.1 & $\mathrm{O} 15-\mathrm{C} 31-\mathrm{H} 31 \mathrm{~B}$ & 109.5 \\
\hline $\mathrm{C} 1-\mathrm{C} 2-\mathrm{H} 2$ & 120.1 & $\mathrm{H} 31 \mathrm{~A}-\mathrm{C} 31-\mathrm{H} 31 \mathrm{~B}$ & 109.5 \\
\hline $\mathrm{C} 2-\mathrm{C} 3-\mathrm{C} 4$ & $119.8(3)$ & $\mathrm{O} 15-\mathrm{C} 31-\mathrm{H} 31 \mathrm{C}$ & 109.5 \\
\hline $\mathrm{C} 2-\mathrm{C} 3-\mathrm{H} 3$ & 120.1 & $\mathrm{H} 31 \mathrm{~A}-\mathrm{C} 31-\mathrm{H} 31 \mathrm{C}$ & 109.5 \\
\hline $\mathrm{C} 4-\mathrm{C} 3-\mathrm{H} 3$ & 120.1 & $\mathrm{H} 31 \mathrm{~B}-\mathrm{C} 31-\mathrm{H} 31 \mathrm{C}$ & 109.5 \\
\hline $\mathrm{C} 5-\mathrm{C} 4-\mathrm{C} 3$ & $118.0(3)$ & & \\
\hline $\mathrm{Cu} 1-\mathrm{O} 7-\mathrm{N} 7-\mathrm{O} 8$ & $172.8(2)$ & $\mathrm{C} 13-\mathrm{C} 14-\mathrm{C} 15-\mathrm{C} 16$ & $178.1(3)$ \\
\hline $\mathrm{Cu} 1-\mathrm{O} 7-\mathrm{N} 7-\mathrm{O} 9$ & $-6.8(4)$ & $\mathrm{C} 20-\mathrm{N} 4-\mathrm{C} 16-\mathrm{C} 17$ & $2.7(5)$ \\
\hline $\mathrm{Cu} 1-\mathrm{O} 11-\mathrm{N} 8-\mathrm{O} 10$ & $-111.9(3)$ & $\mathrm{Cu} 2-\mathrm{N} 4-\mathrm{C} 16-\mathrm{C} 17$ & $-178.7(3)$ \\
\hline $\mathrm{Cu} 1-\mathrm{O} 11-\mathrm{N} 8-\mathrm{O} 12$ & $68.9(3)$ & $\mathrm{C} 20-\mathrm{N} 4-\mathrm{C} 16-\mathrm{C} 15$ & $-178.2(3)$ \\
\hline $\mathrm{Cu} 2-\mathrm{O} 12-\mathrm{N} 8-\mathrm{O} 11$ & $-9.2(3)$ & $\mathrm{Cu} 2-\mathrm{N} 4-\mathrm{C} 16-\mathrm{C} 15$ & $0.4(3)$ \\
\hline $\mathrm{Cu} 2-\mathrm{O} 12-\mathrm{N} 8-\mathrm{O} 10$ & $171.6(2)$ & $\mathrm{N} 3-\mathrm{C} 15-\mathrm{C} 16-\mathrm{C} 17$ & $171.4(3)$ \\
\hline $\mathrm{C} 5-\mathrm{N} 1-\mathrm{C} 1-\mathrm{O} 1$ & $-177.7(3)$ & $\mathrm{C} 14-\mathrm{C} 15-\mathrm{C} 16-\mathrm{C} 17$ & $-8.2(5)$ \\
\hline $\mathrm{Cu} 1-\mathrm{N} 1-\mathrm{C} 1-\mathrm{O} 1$ & $7.1(4)$ & N3-C15-C16-N4 & $-7.7(4)$ \\
\hline $\mathrm{C} 5-\mathrm{N} 1-\mathrm{C} 1-\mathrm{C} 2$ & $1.9(4)$ & $\mathrm{C} 14-\mathrm{C} 15-\mathrm{C} 16-\mathrm{N} 4$ & $172.7(3)$ \\
\hline $\mathrm{Cu} 1-\mathrm{N} 1-\mathrm{C} 1-\mathrm{C} 2$ & $-173.2(2)$ & $\mathrm{N} 4-\mathrm{C} 16-\mathrm{C} 17-\mathrm{C} 18$ & $-3.5(5)$ \\
\hline $\mathrm{O} 1-\mathrm{C} 1-\mathrm{C} 2-\mathrm{C} 3$ & $178.2(3)$ & $\mathrm{C} 15-\mathrm{C} 16-\mathrm{C} 17-\mathrm{C} 18$ & $177.5(3)$ \\
\hline $\mathrm{N} 1-\mathrm{C} 1-\mathrm{C} 2-\mathrm{C} 3$ & $-1.5(5)$ & $\mathrm{C} 16-\mathrm{C} 17-\mathrm{C} 18-\mathrm{C} 19$ & $0.9(5)$ \\
\hline $\mathrm{C} 1-\mathrm{C} 2-\mathrm{C} 3-\mathrm{C} 4$ & $-0.3(5)$ & $\mathrm{C} 17-\mathrm{C} 18-\mathrm{C} 19-\mathrm{C} 20$ & $2.1(5)$ \\
\hline $\mathrm{C} 2-\mathrm{C} 3-\mathrm{C} 4-\mathrm{C} 5$ & $1.7(5)$ & $\mathrm{Cu} 3-\mathrm{O} 4-\mathrm{C} 20-\mathrm{N} 4$ & $19.3(5)$ \\
\hline $\mathrm{C} 1-\mathrm{N} 1-\mathrm{C} 5-\mathrm{C} 4$ & $-0.5(4)$ & $\mathrm{Cu} 3-\mathrm{O} 4-\mathrm{C} 20-\mathrm{C} 19$ & $-160.8(3)$ \\
\hline $\mathrm{Cu} 1-\mathrm{N} 1-\mathrm{C} 5-\mathrm{C} 4$ & $175.1(3)$ & $\mathrm{C} 16-\mathrm{N} 4-\mathrm{C} 20-\mathrm{O} 4$ & $-179.6(3)$ \\
\hline $\mathrm{C} 1-\mathrm{N} 1-\mathrm{C} 5-\mathrm{C} 6$ & $179.7(3)$ & $\mathrm{Cu} 2-\mathrm{N} 4-\mathrm{C} 20-\mathrm{O} 4$ & $2.1(5)$ \\
\hline $\mathrm{Cu} 1-\mathrm{N} 1-\mathrm{C} 5-\mathrm{C} 6$ & $-4.6(3)$ & $\mathrm{C} 16-\mathrm{N} 4-\mathrm{C} 20-\mathrm{C} 19$ & $0.5(5)$ \\
\hline $\mathrm{C} 3-\mathrm{C} 4-\mathrm{C} 5-\mathrm{N} 1$ & $-1.3(5)$ & $\mathrm{Cu} 2-\mathrm{N} 4-\mathrm{C} 20-\mathrm{C} 19$ & $-177.8(2)$ \\
\hline $\mathrm{C} 3-\mathrm{C} 4-\mathrm{C} 5-\mathrm{C} 6$ & $178.5(3)$ & $\mathrm{C} 18-\mathrm{C} 19-\mathrm{C} 20-\mathrm{O} 4$ & $177.2(3)$ \\
\hline $\mathrm{C} 10-\mathrm{N} 2-\mathrm{C} 6-\mathrm{C} 7$ & $0.5(5)$ & $\mathrm{C} 18-\mathrm{C} 19-\mathrm{C} 20-\mathrm{N} 4$ & $-2.9(5)$ \\
\hline $\mathrm{Cu} 1-\mathrm{N} 2-\mathrm{C} 6-\mathrm{C} 7$ & $176.2(2)$ & $\mathrm{C} 25-\mathrm{N} 5-\mathrm{C} 21-\mathrm{O} 5$ & $179.1(3)$ \\
\hline $\mathrm{C} 10-\mathrm{N} 2-\mathrm{C} 6-\mathrm{C} 5$ & $-178.6(3)$ & $\mathrm{Cu} 3-\mathrm{N} 5-\mathrm{C} 21-\mathrm{O} 5$ & $-3.4(5)$ \\
\hline $\mathrm{Cu} 1-\mathrm{N} 2-\mathrm{C} 6-\mathrm{C} 5$ & $-2.8(3)$ & $\mathrm{C} 25-\mathrm{N} 5-\mathrm{C} 21-\mathrm{C} 22$ & $-0.8(5)$ \\
\hline $\mathrm{N} 1-\mathrm{C} 5-\mathrm{C} 6-\mathrm{N} 2$ & $4.9(4)$ & $\mathrm{Cu} 3-\mathrm{N} 5-\mathrm{C} 21-\mathrm{C} 22$ & $176.7(2)$ \\
\hline $\mathrm{C} 4-\mathrm{C} 5-\mathrm{C} 6-\mathrm{N} 2$ & $-174.8(3)$ & $\mathrm{O} 5-\mathrm{C} 21-\mathrm{C} 22-\mathrm{C} 23$ & $-178.6(3)$ \\
\hline $\mathrm{N} 1-\mathrm{C} 5-\mathrm{C} 6-\mathrm{C} 7$ & $-174.1(3)$ & $\mathrm{N} 5-\mathrm{C} 21-\mathrm{C} 22-\mathrm{C} 23$ & $1.4(5)$ \\
\hline
\end{tabular}




$\begin{array}{llll}\mathrm{C} 4-\mathrm{C} 5-\mathrm{C} 6-\mathrm{C} 7 & 6.2(5) & \mathrm{C} 21-\mathrm{C} 22-\mathrm{C} 23-\mathrm{C} 24 & -1.1(5) \\ \mathrm{N} 2-\mathrm{C} 6-\mathrm{C} 7-\mathrm{C} 8 & -0.5(5) & \mathrm{C} 22-\mathrm{C} 23-\mathrm{C} 24-\mathrm{C} 25 & 0.3(5) \\ \mathrm{C} 5-\mathrm{C} 6-\mathrm{C} 7-\mathrm{C} 8 & 178.5(3) & \mathrm{C} 21-\mathrm{N} 5-\mathrm{C} 25-\mathrm{C} 24 & 0.0(5) \\ \mathrm{C} 6-\mathrm{C} 7-\mathrm{C} 8-\mathrm{C} 9 & -0.7(5) & \mathrm{C} 3-\mathrm{N} 5-\mathrm{C} 25-\mathrm{C} 24 & -177.8(3) \\ \mathrm{C} 7-\mathrm{C} 8-\mathrm{C} 9-\mathrm{C} 10 & 1.8(5) & \mathrm{C} 21-\mathrm{N} 5-\mathrm{C} 25-\mathrm{C} 26 & 177.6(3) \\ \mathrm{C} 6-\mathrm{N} 2-\mathrm{C} 10-\mathrm{O} 2 & -178.4(3) & \mathrm{C} 3-\mathrm{N} 5-\mathrm{C} 25-\mathrm{C} 26 & -0.2(4) \\ \mathrm{Cu} 1-\mathrm{N} 2-\mathrm{C} 10-\mathrm{O} 2 & 6.6(5) & \mathrm{C} 23-\mathrm{C} 24-\mathrm{C} 25-\mathrm{N} 5 & 0.2(5) \\ \mathrm{C} 6-\mathrm{N} 2-\mathrm{C} 10-\mathrm{C} 9 & 0.7(5) & \mathrm{C} 23-\mathrm{C} 24-\mathrm{C} 25-\mathrm{C} 26 & -177.2(3) \\ \mathrm{Cu} 1-\mathrm{N} 2-\mathrm{C} 10-\mathrm{C} 9 & -174.3(2) & \mathrm{C} 30-\mathrm{N} 6-\mathrm{C} 26-\mathrm{C} 27 & 2.6(5) \\ \mathrm{C} 8-\mathrm{C} 9-\mathrm{C} 10-\mathrm{O} 2 & 177.3(3) & \mathrm{C} 3-\mathrm{N} 6-\mathrm{C} 26-\mathrm{C} 27 & -173.8(3) \\ \mathrm{C} 8-\mathrm{C} 9-\mathrm{C} 10-\mathrm{N} 2 & -1.8(5) & \mathrm{C} 30-\mathrm{N} 6-\mathrm{C} 26-\mathrm{C} 25 & -175.0(3) \\ \mathrm{Cu} 1-\mathrm{O} 3-\mathrm{C} 11-\mathrm{N} 3 & 67.6(4) & \mathrm{Cu} 3-\mathrm{N} 6-\mathrm{C} 26-\mathrm{C} 25 & 8.6(3) \\ \mathrm{Cu} 1-\mathrm{O} 3-\mathrm{C} 11-\mathrm{C} 12 & -113.4(3) & \mathrm{N} 5-\mathrm{C} 25-\mathrm{C} 26-\mathrm{N} 6 & -5.7(4) \\ \mathrm{C} 15-\mathrm{N} 3-\mathrm{C} 11-\mathrm{O} 3 & 176.9(3) & \mathrm{C} 24-\mathrm{C} 25-\mathrm{C} 26-\mathrm{N} 6 & 171.9(3) \\ \mathrm{Cu} 2-\mathrm{N} 3-\mathrm{C} 11-\mathrm{O} 3 & -12.0(4) & \mathrm{N} 5-\mathrm{C} 25-\mathrm{C} 26-\mathrm{C} 27 & 176.8(3) \\ \mathrm{C} 15-\mathrm{N} 3-\mathrm{C} 11-\mathrm{C} 12 & -2.0(5) & \mathrm{C} 24-\mathrm{C} 25-\mathrm{C} 26-\mathrm{C} 27 & -5.7(5) \\ \mathrm{Cu} 2-\mathrm{N} 3-\mathrm{C} 11-\mathrm{C} 12 & 169.1(2) & \mathrm{N} 6-\mathrm{C} 26-\mathrm{C} 27-\mathrm{C} 28 & -3.2(5) \\ \mathrm{O} 3-\mathrm{C} 11-\mathrm{C} 12-\mathrm{C} 13 & -179.4(3) & \mathrm{C} 25-\mathrm{C} 26-\mathrm{C} 27-\mathrm{C} 28 & 174.2(3) \\ \mathrm{N} 3-\mathrm{C} 11-\mathrm{C} 12-\mathrm{C} 13 & -0.5(5) & \mathrm{C} 26-\mathrm{C} 27-\mathrm{C} 28-\mathrm{C} 29 & 1.0(6) \\ \mathrm{C} 11-\mathrm{C} 12-\mathrm{C} 13-\mathrm{C} 14 & 2.1(5) & \mathrm{C} 27-\mathrm{C} 28-\mathrm{C} 29-\mathrm{C} 30 & 1.6(5) \\ \mathrm{C} 12-\mathrm{C} 13-\mathrm{C} 14-\mathrm{C} 15 & -1.1(5) & \mathrm{C} 26-\mathrm{N} 6-\mathrm{C} 30-\mathrm{O} 6 & 178.8(3) \\ \mathrm{C} 11-\mathrm{N} 3-\mathrm{C} 15-\mathrm{C} 14 & 3.1(5) & \mathrm{Cu} 3-\mathrm{N} 6-\mathrm{C} 30-\mathrm{O} 6 & -5.5(5) \\ \mathrm{Cu} 2-\mathrm{N} 3-\mathrm{C} 15-\mathrm{C} 14 & -168.7(3) & \mathrm{C} 26-\mathrm{N} 6-\mathrm{C} 30-\mathrm{C} 29 & 0.2(5) \\ \mathrm{C} 11-\mathrm{N} 3-\mathrm{C} 15-\mathrm{C} 16 & -176.5(3) & \mathrm{Cu} 3-\mathrm{N} 6-\mathrm{C} 30-\mathrm{C} 29 & 175.9(3) \\ \mathrm{Cu} 2-\mathrm{N} 3-\mathrm{C} 15-\mathrm{C} 16 & 11.7(3) & \mathrm{C} 28-\mathrm{C} 29-\mathrm{C} 30-\mathrm{O} 6 & 179.1(3) \\ \mathrm{C} 13-\mathrm{C} 14-\mathrm{C} 15-\mathrm{N} 3 & -1.5(5) & \mathrm{C} 28-\mathrm{C} 29-\mathrm{C} 30-\mathrm{N} 6 & -2.2(5) \\ & & & \end{array}$

Hydrogen-bond geometry $\left(\AA,{ }^{\circ}\right)$

\begin{tabular}{lllll}
\hline$D-\mathrm{H} \cdots A$ & $D-\mathrm{H}$ & $\mathrm{H} \cdots A$ & $D \cdots A$ & $D-\mathrm{H} \cdots A$ \\
\hline $\mathrm{O} 1-\mathrm{H} 1 \cdots \mathrm{O} 14$ & 0.84 & 1.61 & $2.448(3)$ & 175 \\
$\mathrm{O} 2-\mathrm{H} 2 A \cdots \mathrm{O} 3$ & 0.84 & 1.66 & $2.499(3)$ & 172 \\
$\mathrm{O} 5-\mathrm{H} 5 \cdots \mathrm{O} 13$ & 0.84 & 1.70 & $2.536(3)$ & 170 \\
$\mathrm{O} 6-\mathrm{H} 6 \cdots \mathrm{O} 4$ & 0.84 & 1.67 & $2.495(3)$ & 168 \\
$\mathrm{O} 13-\mathrm{H} 13 A \cdots \mathrm{O} 99^{\mathrm{i}}$ & $0.83(2)$ & $1.98(2)$ & $2.763(3)$ & $158(4)$ \\
$\mathrm{O} 14-\mathrm{H} 14 A \cdots \mathrm{O} 9$ & $0.82(2)$ & $1.94(2)$ & $2.738(3)$ & $163(4)$ \\
$\mathrm{O} 15-\mathrm{H} 15 \cdots \mathrm{O} 10^{\mathrm{ii}}$ & $0.85(5)$ & $2.42(5)$ & $2.790(4)$ & $107(4)$ \\
\hline
\end{tabular}

Symmetry codes: (i) $-x+1,-y+1,-z+1$; (ii) $-x+1,-y,-z+1$. 